\title{
PRIMORDIAL GRAVITATIONAL WAVE DETECTABILITY WITH DEEP SMALL-SKY COSMIC MICROWAVE BACKGROUND EXPERIMENTS
}

\author{
M. Farhang ${ }^{1,2}$, J. R. Bond ${ }^{1}$, O. Doré ${ }^{2,3,4}$, ANd C. B. NetTerfield ${ }^{1,5}$ \\ ${ }^{1}$ Department of Astronomy and Astrophysics, University of Toronto, 50 St. George, Toronto, ON M5S 3H4, Canada \\ ${ }^{2}$ Canadian Institute for Theoretical Astrophysics, 60 St. George, Toronto, ON M5S 3H8, Canada \\ ${ }^{3}$ Jet Propulsion Laboratory, California Institute of Technology, Pasadena, CA 91109, USA \\ ${ }^{4}$ California Institute of Technology, Pasadena, CA 91125, USA \\ ${ }^{5}$ Department of Physics, University of Toronto, 60 St. George Street, Toronto, ON M5S 1A7, Canada \\ Received 2011 August 7; accepted 2013 April 22; published 2013 June 10
}

\begin{abstract}
We use the Bayesian estimation on direct $T-Q-U$ cosmic microwave background (CMB) polarization maps to forecast errors on the tensor-to-scalar power ratio $r$, and hence on primordial gravitational waves, as a function of sky coverage $f_{\text {sky. }}$. This map-based likelihood filters the information in the pixel-pixel space into the optimal combinations needed for $r$ detection for cut skies, providing enhanced information over a first-step linear separation into a combination of $E, B$, and mixed modes, and ignoring the latter. With current computational power and for typical resolutions appropriate for $r$ detection, the large matrix inversions required are accurate and fast. Our simulations explore two classes of experiments, with differing bolometric detector numbers, sensitivities, and observational strategies. One is motivated by a long duration balloon experiment like Spider, with pixel noise $\propto \sqrt{f_{\text {sky }}}$ for a specified observing period. This analysis also applies to ground-based array experiments. We find that, in the absence of systematic effects and foregrounds, an experiment with Spider-like noise concentrating on $f_{\text {sky }} \sim 0.02-0.2$ could place a $2 \sigma_{r} \approx 0.014$ boundary ( $~ 95 \%$ confidence level), which rises to 0.02 with an $\ell$-dependent foreground residual left over from an assumed efficient component separation. We contrast this with a Planck-like fixed instrumental noise as $f_{\text {sky }}$ varies, which gives a Galaxy-masked $\left(f_{\text {sky }}=0.75\right) 2 \sigma_{r} \approx 0.015$, rising to $\approx 0.05$ with the foreground residuals. Using as the figure of merit the (marginalized) one-dimensional Shannon entropy of $r$, taken relative to the first 2003 WMAP CMB-only constraint, gives -2.7 bits from the 2012 WMAP9+ACT+SPT+LSS data, and forecasts of -6 bits from Spider (+ Planck); this compares with up to -11 bits for CMBPol, COrE, and PIXIE post-Planck satellites and -13 bits for a perfectly noiseless cosmic variance limited experiment. We thus confirm the wisdom of the current strategy for $r$ detection of deeply probed patches covering the $f_{\text {sky }}$ minimum-error trough with balloon and ground experiments.
\end{abstract}

Key words: cosmic background radiation - cosmological parameters - cosmology: theory - methods: numerical

Online-only material: color figures

\section{INTRODUCTION}

Inflation, a period of accelerated expansion in the very early universe, is the most widely accepted scenario to solve the problems of the otherwise successful standard model of cosmology. The so-called $B$-mode of the cosmic microwave background $(\mathrm{CMB})$ polarization provides cosmologists with a unique opportunity to test the theory of inflation and to probe its high energy scales. In the rest of this Introduction, the basics of the theory of inflation is briefly reviewed (Section 1.1) and the current observational status of $B$-mode detection is discussed (Section 1.2). At the end, the framework of the paper and our proposed approach in the analysis of CMB polarization with the goal of inflation-induced $B$-mode detection are presented.

\subsection{Inflation and Its Observables}

In the simplest models of inflation, the postulated accelerated expansion in the early universe is driven by an effective potential energy $V(\phi)$ of a single scalar field degree of freedom $\phi$ (see, e.g., Dodelson 2003; Mukhanov 2005; Weinberg 2008),

$$
\begin{aligned}
H^{2} & =\frac{1}{3 M_{\mathrm{P}}^{2}}\left(\frac{\dot{\phi}^{2}}{2}+V(\phi)\right) \\
\frac{\ddot{a}}{a} & =-\frac{1}{3 M_{\mathrm{P}}^{2}}\left(\dot{\phi}^{2}-V(\phi)\right) .
\end{aligned}
$$

The evolution of the scalar field is described by

$$
\ddot{\phi}+3 H \dot{\phi}+V^{\prime}(\phi)=0 \text {. }
$$

An unavoidable consequence of inflation is the generation of scalar and tensor zero-point fluctuations in the spacetime metric (Mukhanov \& Chibisov 1981; Hawking 1982; Starobinsky 1982; Bardeen et al. 1983; Kodama \& Sasaki 1984; Guth \& Pi 1985). The former are curvature perturbations, with associated density fluctuations that can grow via gravitational instability to create the cosmic web, with its rich observational characterization. The latter are gravity waves that induce potentially observable signatures in the spatial structure of the $\mathrm{CMB}$, in particular in its polarization, the focus of this paper. Whereas curl-free $E$-modes of polarization can be produced both by tensor and scalar perturbations, divergence-free modes of $\mathrm{CMB}$ polarization ( $B$-modes) would be induced on large scales by primordial gravitational waves but not by scalar curvature fluctuations. At smaller scales, $B$-modes are induced from primordial $E$-modes through gravitational lensing distortions of the CMB polarization patterns (Zaldarriaga \& Seljak 1998), adding to the complexity of making a clean separation of the tensor-induced signal.

The primordial scalar and tensor power spectra (fluctuation variances per $\ln k$ ) and their ratio $r(k)$ are often approximated by power laws in the three-dimensional (3D) comoving 
wavenumber $k$,

$$
\begin{aligned}
\mathcal{P}_{\mathrm{s}}(k) & \approx A_{\mathrm{s}}\left(k_{\mathrm{sp}}\right)\left(k / k_{\mathrm{sp}}\right)^{n_{\mathrm{s}}\left(k_{\mathrm{sp}}\right)-1}, \\
\mathcal{P}_{\mathrm{t}}(k) & \approx A_{\mathrm{t}}\left(k_{\mathrm{tp}}\right)\left(k / k_{\mathrm{tp}}\right)^{n_{\mathrm{t}}\left(k_{\mathrm{tp}}\right)}, \\
r(k) & \equiv \mathcal{P}_{\mathrm{t}}(k) / \mathcal{P}_{\mathrm{s}}(k) \approx r\left(k / k_{\mathrm{tp}}\right)^{n_{\mathrm{t}}\left(k_{\mathrm{tp}}\right)-n_{\mathrm{s}}\left(k_{\mathrm{tp}}\right)+1}, \\
r & \equiv r\left(k_{\mathrm{tp}}\right) \equiv \mathcal{P}_{\mathrm{t}}\left(k_{\mathrm{tp}}\right) / \mathcal{P}_{\mathrm{s}}\left(k_{\mathrm{tp}}\right),
\end{aligned}
$$

where the normalization factors $A_{\mathrm{s}}\left(k_{\mathrm{sp}}\right)$ and $A_{\mathrm{t}}\left(k_{\mathrm{tp}}\right)$ are the amplitudes of the scalar and tensor power spectra at the pivots $k_{\mathrm{sp}}$ and $k_{\mathrm{tp}}$, respectively. The pivots $k_{\mathrm{sp}}$ and $k_{\mathrm{tp}}$ about which the expansions occur are usually chosen to be different for scalars and tensors to reflect where the optimal signal weights come from. The main target of many of the current and coming CMB polarization experiments is, first, a one-parameter uniform $r$. An advantage of this ratio over $\mathcal{P}_{\mathrm{t}}\left(k_{\mathrm{tp}}\right)$ is that it removes a dominant near-degeneracy with the Thompson depth to Compton scattering $\tau$. The trajectory $r(k)$ also measures the inflation acceleration history $\epsilon(a) \equiv-d \ln H / d \ln a$. Note that in $r(k), n_{\mathrm{s}}$ is approximated by $n_{\mathrm{s}}\left(k_{\mathrm{tp}}\right)$, i.e., at a different pivot, assuming small running of $n_{\mathrm{s}}$ with wavenumber. To the first order in the slow-roll parameter $\epsilon, r(k)$ can be directly related to $V$ through the relation

$$
\begin{aligned}
r(k) & \approx 16 \epsilon,(a \approx k / H) \\
V & \approx \frac{3 \pi^{2}}{2} M_{\mathrm{P}}^{4} r \mathcal{P}_{\mathrm{s}} \sim\left(10^{16} \mathrm{GeV}\right)^{4} r / 0.008 .
\end{aligned}
$$

We have used $10^{10} A_{\mathrm{s}} \approx 24.4{ }^{6}$ Here $M_{\mathrm{P}}=1 / \sqrt{8 \pi G}$ is the reduced Planck mass, with $c$ and $\hbar$ set to unity. The relation $k \approx H a$ of resolution $k^{-1}$ to the dynamics encoded in the expansion and Hubble parameters, $a$ and $H$, is only approximate of course, but very useful (e.g., Bond 1996).

There is no consensus on what pure theory will tell us about the best value for $r$, or even its likely range. However, if $r$ drops below the benchmark $r \sim 0.01$ set by the GUT scale $\sim 10^{16} \mathrm{GeV}$, the consequences will be profound (Baumann et al. 2009). Experiments with Spider-like sensitivity could probe such a limit. In this paper, we explore the very small $r_{\text {fid }}<0.01$ regime. To show what happens when there are detections, we often use $r_{\text {fid }}=0.12$ as a fiducial high- $r$ case for tests, a value which lies just below the 0.13 coming from the simplest $V=m^{2} \phi^{2} / 2$ chaotic inflation model (Linde 1983).

In addition to the deviations of the slopes from scale invariance $\left(n_{\mathrm{t}}=0\right.$ and $\left.n_{\mathrm{s}}-1=0\right)$, the slopes are expected to "run with $k$," although they may be approximately constant over the observable CMB range. In this paper $n_{\mathrm{s}}(k)$ is not our target, nor are high multipole CMB experiments which are necessary to get the long baseline needed to show whether or not $n_{\mathrm{s}}$ runs.

A consequence of the fall-off of the tensor-induced CMB signal beyond $\ell \sim 150$ is that only limited information can be obtained on $n_{\mathrm{t}}(k)$ - enough to allow a number of broad bands for $r(k)$, but not enough for $n_{\mathrm{t}}\left(k_{\mathrm{tp}}\right)$, let alone $n_{\mathrm{t}}(k)$, to be determined with sufficient accuracy to test well the inflation consistency relation for gravity waves. In the limited two-parameter tensor parameter space of $r$ and uniform $n_{\mathrm{t}}$, this consistency condition to the first order of the slow roll parameters is (e.g., Kolb \& Turner 1990)

$$
n_{\mathrm{t}} \approx-\frac{r}{8}
$$

\footnotetext{
6 http://lambda.gsfc.nasa.gov/product/map/dr4/params/lcdm_sz_lens_ wmap7_bao_h0.cfm
}

so a convincing test would require an order of magnitude better determination of $n_{\mathrm{t}}$ than $r$.

Another complication in relating the experiments to inflation theory is that there is still observational room for subdominant scalar isocurvature perturbations in addition to the dominant curvature ones when multiple fields are dynamically important during or immediately after inflation (Linde 1984; Efstathiou \& Bond 1986; Kodama \& Sasaki 1986; Kofman \& Linde 1987; Peebles 1987). Isocurvature perturbations with a nearly scale invariant primordial spectrum have significantly enhanced low- $\ell$ CMB power (e.g., Bond 1996), and that region, overlapping with the gravity wave-induced CMB power, is where the constraint on the overall isocurvature amplitude comes from (Sievers et al. 2007 and Larson et al. 2011).

\subsection{Observations}

All CMB polarization experiments are limited in sky coverage by instrumental or Galactic foreground constraints. Thus, even though the $B$-modes provide a unique $r$-signature and are orthogonal to the $E$-modes over the full sky, realistically mode mixing must always be dealt with, even though it may be larger for smaller $f_{\text {sky }}$. Assessing the tradeoffs between shallow large-sky and deep small-sky observational strategies is the target of our investigation. Going for deep and small has the advantage that one can select the most foreground-free patches to target to decrease the high level of foreground subtraction. In addition, the long waves which dominate foregrounds are naturally filtered. Ground-based or balloon-borne experiments using the deep and small-sky strategy include BICEP (Chiang et al. 2010) and BICEP2, ${ }^{7}$ QUIET $^{8}$ (QUIET Collaboration et al. 2010), PolarBear' (The PolarBear Collaboration et al. 2010), EBEX $^{10}$ (Reichborn-Kjennerud et al. 2010), Spider ${ }^{11}$ (Fraisse et al. 2013), KECK (Sheehy et al. 2010), ABS ${ }^{12}$ (EssingerHileman et al. 2010), PIPER (Chuss et al. 2010). Planck (and $W M A P)$ are (relatively) shallow and large-sky. Proposed nextgeneration satellite experiments such as COrE (The COrE Collaboration 2011), PIXIE (Kogut et al. 2011) and LiteBIRD ${ }^{13}$ are deep and large-sky.

The current $2 \sigma \quad r$-constraints come from the CMB-only data from ACT+SPT $+W M A P 9(<0.17)$ and with LSS added $(<0.12)$ (Hinshaw et al. 2012). Figure 1 gives a succinct summary of the current status of $r$ measurements and what can be achieved with the Spider-like sensitivities we use as an example throughout the text, compared to a case of Spider with more realistic specifications as envisaged in Fraisse et al. (2013) (labeled as "Spider" in the plot), and for an even more ambitious campaign of subsequent flights of the Spider instrument, as proposed for SCIP. It also shows an idealized CMBPol allsky experiment like PIXIE or COrE. The various theoretical possibilities shown for $r$ are swept through by these achievable (foreground-less) $r$-likelihood curves. The main purpose of this paper is to explain how these forecasted likelihoods are obtained.

In this paper, we first review the general Bayesian framework for determining parameters to introduce the notations we use. We cast the quest for $r$ into an information-theoretic language

\footnotetext{
7 http://bicep.caltech.edu/public/

8 http://quiet.uchicago.edu/

9 http://bolo.berkeley.edu/polarbear/

10 http://groups.physics.umn.edu/cosmology/ebex/

11 http://www.astro.caltech.edu/ lgg/spider/spider_front.htm

12 http://www.princeton.edu/physics/research/cosmology-experiment/ abs-experiment/

13 http://cmbpol.kek.jp/litebird/index.html
} 


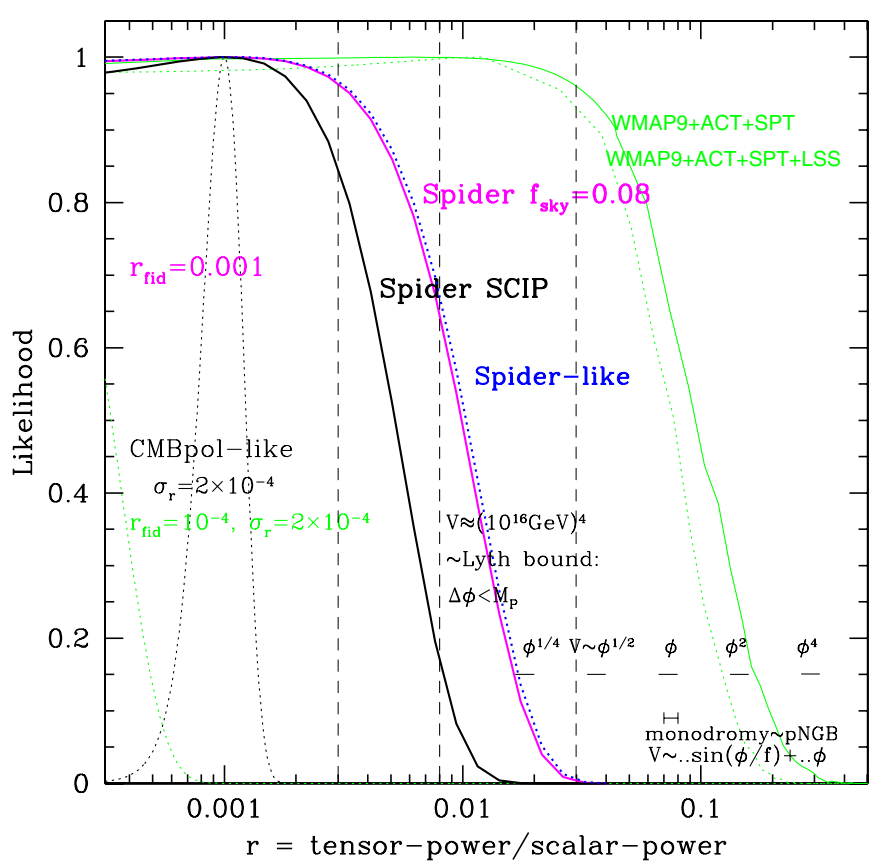

Figure 1. Forecasted $r$-likelihood values for the Spider-like specifications used in the paper observing $8 \%$ of the sky. The one labeled Spider corresponds to the actual, more recent Spider proposal with two flights described in (Fraisse et al. 2013, see the footnotes of Table 1). The SCIP envisages three subsequent flights of the Spider payload. This is contrasted with the current constraints from the $\mathrm{ACT}+\mathrm{SPT}+W M A P 9$ data, and also combined with the measurements of LSS (Hinshaw et al. 2012). The marginalized 1D likelihood curves are based on the publicly available chains (http://lambda.gsfc.nasa.gov/ product/act/act_chainsv2_get.cfm) binned into 50 bins, and Gaussian-fitted to plot the very small $r$ region where not enough points were available. These are compared with a forecast for an idealized $C M B P o l$ all-sky experiment like PIXIE or COrE with a $\sigma_{r} \sim 0.0002$ error. A model input of $r_{\text {fid }}=0.001$ was assumed, which would give a solid $C M B P o l$ detection for it. A $r_{\text {fid }}=0.0001$ case is also shown. Foregrounds and systematic errors were ignored in these plots; modifications resulting from errors in foreground subtractions are shown in other plots in the paper. A number of theoretical predictions are also shown, for power law inflation potentials with slope ranging from 0.25 up to 4 . The width covers the range of 60 to $50 e$-folds for inflation. The linear potential is contrasted with the similar range for a string-inspired mixed model called monodromy (McAllister et al. 2010) with a linear potential added to a sinusoidal pseudo-Nambu-Goldstone potential. A few target lines are also shown, one at 0.03 which many theories of the 1980s and 1990s were above, one at 0.008 corresponding to an inflation energy scale about the grand unification scale, which is near the Lyth boundary (Lyth 1997) as indicated. Supergravity-inspired theories can obtain values anywhere in the range from $\sim 0.003$ to $\sim 0.3$ (Kallosh \& Linde 2010). Thus, small-patch experiments with Spider-like specifications could explore much of the $r$-terrain of theoretical relevance.

(A color version of this figure is available in the online journal.)

in which the forecasted outcomes of different experiments can be contrasted by considering the differences in their reduced a posteriori Shannon entropies for $r, S_{1 \mathrm{f}}(r \mid \mathrm{expt})$. We discuss the two basic approaches for constraining cosmological observables, such as those associated with inflation, and the relation of these to $E-B$ mixing: (1) the $\ell$-space approach in which $\mathrm{CMB}$ maps are first compressed onto power spectrum parameters for $T T-T E-E E$ and $B B$, which are then compressed onto cosmic parameters; and (2) direct parameter extraction of $r$ from map likelihoods. Our primary target is $r$ and not the $B$-mode spectrum; hence the optimal one-step estimation from maps is preferred, provided it is computationally feasible-which it is for Spider-like experiments. The leakage between the $E$ and $B$ modes and its impact on $r$ is quantified in Section 3. In Section 4, we present details of the method we use to bypass explicit $E-B$ de-mixing and apply it to simulated data for realistic in- strumental and foreground-residual noise levels for Spider-like and Planck-like experiments as $f_{\text {sky }}$ varies. We end with our conclusions from this study.

\section{BAYESIAN CMB ANALYSIS OF BANDPOWERS AND COSMIC PARAMETERS}

As has become conventional in CMB analysis, the framework envisaged to compress the information from Spider-like raw time-ordered data to constraints on cosmic parameters, in particular our target $r$, is one of a long Bayesian chain of conditional probabilities (Bond 1996; Bond \& Crittenden 2001). It includes reducing noisy data to maps, maps to band-powers and then to cosmic parameters, or directly maps to parameters. Starting from pixel maps, we review the framework with polarization to introduce our notation. We also remark on how the associated conditional Shannon entropies decrease as the maps are reduced to a precious set of parameter bits.

\subsection{Pixel Maps and E-B Maps}

The map data vector $\Delta$ for CMB experiments is composed of a number of signals $s$ as well as the map noise $n$. The noise encompasses true instrumental noise, experimental systematic effects, and possibly, may draw terms from the signal side that are unwanted residuals on the sky, e.g., from foreground subtraction uncertainty. Each signal has a frequency dependence and polarization components, labeled by four Stokes parameters $x=T, Q, U, V$ referring to a fixed polarization sky reference frame in real space. The map components for each $x$, each pixel $p=1, \ldots, N_{\text {pix }}$, and each frequency channel $c$ are expressible as

$$
\Delta_{c x p}=\sum_{J} s_{J c x p}+n_{c x p}, \quad x \in\{T, Q, U, V\} .
$$

For Thompson scattering anisotropies, the $V$ Stokes parameter associated with circular polarization vanishes, as it also does for most Galactic foregrounds contaminating the primary CMB signal, so we now drop it from our consideration. It would of course be of interest to show experimentally that there is indeed no circular polarization in the CMB data (e.g., see Cooray et al. 2003).

In the standard expansion of the CMB temperature and polarization fields on an orthogonal basis, the mode functions are the spherical harmonics, spin-0 for $T$, and spin-2 for polarization, and the coefficients $a_{J \nu \ell m}$ are the spherical harmonic signal amplitudes, where $v$ represents the frequency

$$
\begin{aligned}
T_{J v}(\theta, \phi) & =\sum_{\ell=2}^{\infty} \sum_{m=-\ell}^{\ell} a_{J v T \ell m} Y_{\ell m}(\theta, \phi), \\
(Q \pm i U)_{J v}(\theta, \phi) & =\sum_{\ell=2}^{\infty} \sum_{m=-\ell}^{\ell}{ }_{ \pm 2} a_{J \nu \ell m}\left[{ }_{ \pm 2} Y_{\ell m}(\theta, \phi)\right] .
\end{aligned}
$$

The transformation from this natural multipole space for the signals to the map space, $s_{J c x p}$, includes beam information, the frequency response function for the channels, and the mask, whether a sharp cookie cutter or a tapered one.

Further linear combinations of the spin-2 expansion coefficients define the $E$ and $B$ modes:

$$
\begin{aligned}
& a_{J \nu E \ell m}=-\frac{1}{2}\left({ }_{2} a_{J \nu \ell m}+{ }_{2} a_{J \nu \ell m}\right), \\
& a_{J \nu B \ell m}=-\frac{1}{2 i}\left({ }_{2} a_{J \nu \ell m}-{ }_{-2} a_{J \nu \ell m}\right) .
\end{aligned}
$$


The separation of CMB polarization into $E$ and $B$-modes is useful because scalar perturbations only result in the $E$ mode whereas tensor perturbations generate both (Kamionkowski et al. 1997; Zaldarriaga \& Seljak 1997). Nonlinear transport effects associated with the weak lensing of primary CMB fluctuations turn some scalar $E$-mode into scalar $B$-mode, mostly at higher $\ell$ s than given by the tensor component (Zaldarriaga $\&$ Seljak 1998), so separation for $r$ detection can be done. Note that this lensing source has non-Gaussian features, which means the power spectra are not enough to characterize its signal (Zaldarriaga 2000; Okamoto \& Hu 2002; Cooray \& Kesden 2003).

\subsection{Maps to Parameters with Map-based Likelihoods}

Following the familiar Bayesian analysis techniques applied to CMB data (e.g., Bond 1996), we wish to construct the a posteriori probability distribution $P(\mathbf{q} \mid \Delta, \mathcal{T})$ of parameters $\mathbf{q}=\left(q_{1}, \ldots, q_{n}\right)$, an update from the a priori probability $P(\mathbf{q} \mid \mathcal{T})$ on the theory space $\mathcal{T}$ of the parameters that is driven by the likelihood of the data $\Delta$ given $\mathbf{q}, \mathcal{L}_{\Delta}(\mathbf{q}) \equiv P(\Delta \mid \mathbf{q}, \mathcal{T})$,

$$
P(\mathbf{q} \mid \Delta, \mathcal{T})=P(\Delta \mid \mathbf{q}, \mathcal{T}) P(\mathbf{q} \mid \mathcal{T}) / P(\Delta \mid \mathcal{T}) .
$$

The prior may include theoretical prejudice, information derived from other data, and, at the very least, the specific measure adopted for the parameters. The evidence, $P(\Delta \mid \mathcal{T})$, a single normalization, is also needed to ensure that the posterior integrates to unity. Its determination is generally computationally intense if one integrates over all parameter space, but it may only be needed at late stages of reduction, e.g., over 2D and 1D reduced parameter spaces.

If the noise is Gaussian with a covariance matrix $\mathbf{C}_{\mathrm{n}}$ and the signals are also Gaussian with their own covariance $\mathbf{C}_{\mathrm{s}}$ about a zero mean, then

$$
\ln \mathcal{L}_{\Delta}(\mathbf{q})=-\frac{1}{2} \Delta^{\mathrm{T}} \mathbf{C}_{\mathrm{t}}^{-1} \Delta-\frac{1}{2} \ln \operatorname{det} \mathbf{C}_{\mathrm{t}}-\frac{1}{2} N_{\text {pix }} \ln (2 \pi),
$$

where $\mathbf{C}_{\mathrm{t}}=\mathbf{C}_{\mathrm{n}}+\sum_{J J^{\prime}} \mathbf{C}_{\mathrm{s}, J J^{\prime}}$, with

$$
\begin{aligned}
\mathbf{C}_{\mathrm{n}, c x p, c^{\prime} x^{\prime} p^{\prime}} & =\left\langle n_{c x p} n_{c^{\prime} x^{\prime} p^{\prime}}\right\rangle \\
\mathbf{C}_{\mathrm{s}, J c x p, J^{\prime} c^{\prime} x^{\prime} p^{\prime}} & =\left\langle s_{J c x p} s_{J^{\prime} c^{\prime} x^{\prime} p^{\prime}}\right\rangle .
\end{aligned}
$$

We have assumed no correlation between signal and noise.

The extra ingredient needed to determine the posterior $P(\mathbf{q})$ $\Delta, \mathcal{T})$ is the prior defining the measure on $\mathbf{q}, \mathcal{L}_{\text {prior }}(\mathbf{q}) \equiv P(\mathbf{q} \mid$ $\mathcal{T})$. The prior is most often taken to be uniform within some parameter region. Another simple possibility is a Gaussian prior with correlation matrix $F_{\text {prior }}^{-1}$ about the mean $\overline{\mathbf{q}}$. In this work we usually assume the uniform prior, though sometimes for small coverage experiments we use a Gaussian prior reflecting the WMAP determination of parameters.

In this paper we usually determine the full likelihood $\ln \mathcal{L}_{\mathrm{t}} \equiv$ $\ln \left(\mathcal{L}_{\Delta}+\mathcal{L}_{\text {prior }}\right)$ on a suitably chosen grid in the parameter space. We can then search for the maximum likelihood $\mathbf{q}_{\mathrm{m}}$ and, with suitable marginalizations over other variables, obtain 2D significance contours and 1D Bayesian errors. It is customary to refer to methods which directly search for the maximum likelihood point as (map- or pixel-based) maximum likelihood estimators.

\subsection{Bandpower Estimation}

For statistically isotropic signals there are generally six crossspectra among the coefficients,

$$
\begin{aligned}
& \left\langle a_{x \ell m} a_{x^{\prime} \ell^{\prime} m^{\prime}}^{*}\right\rangle=C_{X \ell} \delta_{\ell \ell^{\prime}} \delta_{m m^{\prime}}, X=x x^{\prime}, \\
& \text { for } x \in\{T, E, B\}, X \in\{T T, E E, B B, T E, T B, E B\} .
\end{aligned}
$$

Typically the $E B$ and $T B$ power vanish (theoretically anyway) and only four power spectra are needed to characterize the CMB temperature and polarization fields. However, $E B$ and $T B$ may be kept for systematics monitoring. For statistically homogeneous and isotropic 3D Gaussian initial conditions, the primary CMB $T, Q, U$ are isotropic 2D Gaussian fields whose probability distribution depends only upon the power spectra $C_{X \ell}$, or, equivalently the $X$-power per $\ln (\ell+1 / 2)$,

$$
\mathcal{C}_{X \ell} \equiv \frac{\ell(\ell+1)}{2 \pi} C_{X \ell}
$$

The goal of bandpower estimation is to radically compress the map information onto $\ell$-bandpower amplitudes $q_{X \beta}$, with templates of the form $\mathcal{C}_{X \beta, X \ell}$. With sufficiently fine $\ell$-space banding, this stage of compression can be relatively lossless, allowing the cosmic parameters to be derived accurately. The interband shape of these templates may be crafted to look like theoretically expected shapes, or could just be flat, which imposes no prior prejudice. Both approaches have been effectively used (see, e.g., Myers et al. 2003; Story et al. 2012; Dunkley et al. 2013).

With cut-sky maps, bands are coupled even though they would not be for full sky observations with statistically homogeneous noise. The optimal method for estimating power spectra in the general case is the computationally expensive brute-force maximum likelihood analysis (e.g., Bond et al. 1998). This method iteratively corrects a quadratic expression for deviations of the various bandpowers $q_{\beta}$ from their initial values until the maximum likelihood $q_{\mathrm{m}}^{\beta}$ is reached. The weight matrix $\mathbf{C}_{t}^{-1}(\mathbf{q})$ is adjusted at each step, until it settles into $\mathbf{C}_{t}^{-1}\left(\mathbf{q}_{\mathrm{m}}\right)$. The weight enters in two ways; one is quadratically in the likelihoodcurvature matrix (approximately the Fisher matrix) and the other is in the force that drives the relaxation of the parameters to $q_{\mathrm{m}}^{\beta}$.

These map-based methods for bandpower estimation were used by BOOMERanG (de Bernardis et al. 2000; Ruhl et al. 2003) and in all CBI papers (e.g., Sievers et al. 2003; Myers et al. 2003; Sievers et al. 2009). If bandpowers are linear in the cosmic parameter of interest, like $r$, then the parameter can be viewed as a single-template big-band bandpower. Even with the fully nonlinear $\mathcal{C}_{X \ell}(q)$, the amplitudes $\delta q$ can be iteratively solved for using linear derivative templates, and, with convergence, the result is the same as given a full nonlinear treatment.

\subsection{Pseudo- $C_{X \ell}$ cf. Map-based Methods}

Several fast sub-optimal approximate methods have been developed to make the bandpower computation less computationally intense than in the map-based method, e.g., pseudo- $C_{\ell}$ estimators (Hansen \& Górski 2003; Chon et al. 2004), SPICE (Szapudi et al. 2001), MASTER (Hivon et al. 2002) and Xfaster (C. R. Contaldi et al. 2010, in preparation; Rocha et al. 2010, 2011). Pseudo- $C_{\ell}$ 's are constructed by direct spherical harmonic transform of the cut-sky maps, or more generally, taperweighted CMB maps. The all-sky bandpower centered on a specific $\ell_{\beta}, q_{X \beta}$, is then related to the desired $\ell_{\beta}$-band by an appropriate filtering which draws the pseudo- $C_{X \ell}$ 's from a wide swath of $\ell$ 's determined by a mask-defined coupling matrix. In spite of this $\ell$-space mixing, extensive testing has shown these methods to be accurate for temperature anisotropies for large pixel numbers where the matrix inversions of the iterated quadratic approach are prohibitively expensive computationally. They have also been applied effectively to polarization data sets such as BOOMERanG (Montroy et al. 2006; Piacentini et al. 2006). 
The pseudo- $C_{X} \ell$ 's for $X=E E, B B$ suffer from $E-B$ mixing in addition to the $\ell$-space mixing: the estimated $C_{B B \ell}$ receives contributions from both $E$ and $B$ modes. The contamination coming from the $E$ mode can be removed from $C_{B B \ell}$ in the mean by having the estimators undergo a de-biasing step. However, there is still an extra contribution to the variance of estimators, which is due to the dominance of the relatively large $E$ signal mixed into the $B$ measurement. This can limit the primordial gravitational wave detection to $r \approx 0.05$ for deep small sky surveys (covering about $1 \%$ of the sky) as shown by Challinor $\&$ Chon (2005). Lewis et al. (2002) show how to construct window functions that cleanly separate the $E$ and $B$ modes in harmonic space for azimuthally symmetric sky observations at the cost of some information loss due to the boundary of the patch. In another treatment of the $E-B$ mixing problem, Bunn et al. (2003) show that the polarization maps can be optimally decomposed into three orthogonal components: pure $E$, pure $B$, and ambiguous modes. The ambiguous modes receive a non-restorable contribution from both $E$ and $B$ signals, and are dominated by $E$ signal, and thus should be removed in $B$-mode analysis. Based on this decomposition, a near-optimal pure pseudo- $C_{\ell}$ estimator was proposed (Smith 2006) and developed (Smith \& Zaldarriaga 2007; Grain et al. 2009) which ensures no $E-B$ mixing. Recently Bunn (2011) has given a more efficient recipe for decomposing polarization data into $E, B$ and ambiguous maps, although still along the lines of Bunn et al. (2003).

It is clear that if the full map-likelihood analysis can be done, then it should be done, since relevant information is not being thrown away. There are two drawbacks to this map-based approach. The first is that $\mathbf{C}_{t}$ should saturate all contributions to signal and noise since we are in quest of a small, essentially perturbative, component associated with $r$ whose values can be biased by the missing components. This could be challenging in the presence of complex filtering resulting from time-ordered data processing. Also the computational cost of the required large matrix manipulations is high compared to the suboptimal methods. The matrix size depends upon the fraction of sky covered and the resolution. For example, for an experiment covering $25 \%$ of the sky analyzed at a Healpix resolution of $N_{\text {side }}=64$, the sizes are $35 \mathrm{~K} \times 35 \mathrm{~K}$ and we find that the likelihood calculation takes about 5 minutes on a node with 16 Dual-Core Power 6 CPUs at $4.7 \mathrm{GHz}$ (and theoretically capable of doing 600 GFLOPS/node). In practice, our matrices are usually smaller than this since the quest for $r$ requires a relatively low resolution analysis. Also, only a few other parameters that are correlated with $r$ need to be carried along (see Section 4.3). To include many more parameters, standard Bayesian sampling algorithms such as MCMC and adaptive importance sampling (Wraith et al. 2009) can be used. If we need to cover small angular scales as well as large, the matrices become prohibitively large, and hybrid methods, with a mapbased likelihood for large scales joined to an $\ell$-space-based likelihood for small scales, are needed (see, e.g., Hinshaw et al. 2007).

\subsection{The Downward Flow of Shannon Entropy from Maps to Theory Subspaces}

The Shannon entropy $S_{\mathrm{f}}$ of the final (posterior) probability distribution is an average of the log of the local phase space volume $\left\langle\ln p_{\mathrm{f}}^{-1}\right\rangle_{\mathrm{f}}$ over the posterior probability distribution $p_{\mathrm{f}}$, and is considered to provide an estimate of the total information content in the final ensemble (see, e.g., MacKay 2003),

$$
S_{\mathrm{f}}(\mathcal{T} \mid \mathcal{D})=\left\langle\ln P(\mathbf{q} \mid \mathcal{D}, \mathcal{T})^{-1}\right\rangle_{\mathrm{f}}=-\int d^{N} \mathbf{q} p_{\mathrm{f}} \ln p_{\mathrm{f}},
$$

where $\mathcal{D}$ represents data (here $\mathrm{CMB}$ maps $\Delta$ ). The initial entropy is similarly averaged over the initial ensemble $S_{\mathrm{i}} \equiv$ $\left\langle\ln P(\mathbf{q} \mid \mathcal{T})^{-1}\right\rangle_{\mathrm{i}}$. For a uniform prior over a volume $V_{\mathbf{q}, \mathrm{i}}$ in $\mathbf{q}$-space, it is $S_{\mathrm{i}}=\ln V_{\mathbf{q}, \mathrm{i}}$. The final entropy can be thought of as having a contribution from (the log of) an effective phase space volume, reduced relative to the initial one because of the measurement, plus a term related to the average $\chi^{2}$ associated with the mean-squared deviations of $\mathbf{q}$. This term is usually just the number of degrees of freedom unless the model is a very poor representation of the information content of the data.

It should not seem curious to say that the information entropy decreases as a result of measurements, but it may seem curious to word it as follows: the average information content decreases. That is because the fully random initial state has more information, in that the variables can take on a wider range of values. We think that the reduced post-experiment information content is of higher quality. What constitutes quality in information is of course subjective.

The common figure of merit for error in parameter (here $r$ ) measurement is $2 \sigma_{r}$. That is also what we primarily quote in this paper, determined as explained in Section 2.6. However, a better figure of merit than $2 \sigma_{r}$ is the change in 1D Shannon entropy, which tells us the average amount by which the log of the allowed volume in the $r$ parameter space shrinks in response to varying the experimental setups. It is $1 \mathrm{D}$ because we marginalize over all other $N-1$ parameters, the cosmic ones of interest and any nuisance parameters deemed necessary for the analysis, such as those characterizing uncertainties in calibration, beams, bolometer $T-Q-U$ leakage, and foreground uncertainties.

The 1D Shannon information entropy, $S_{1 \mathrm{f}}(r)=\left\langle\mathcal{S}_{1 \mathrm{I}}(r)\right\rangle_{\mathrm{f}}+$ $\ln P(\Delta \quad \mid \mathcal{T})$, where the information action $\mathcal{S}_{1 \mathrm{I}}(r)_{\mathrm{f}}=$ $-\ln P(\Delta \mid r, \mathcal{T})-\ln (r \mid \mathcal{T})$, is best calculated by numerical integration over the $r$-grid. The result is very simple if we truncate the ensemble-averaged expansion of $\mathcal{S}_{1 \mathrm{I}}(r)$ at quadratic order

$$
S_{1 \mathrm{f}}(r) \approx \frac{1}{2}+\frac{1}{2} \ln (2 \pi)+\ln \left(\sigma_{r}\right)=\frac{1}{2}+\ln V_{r},
$$

where $V_{r}$ (defined by the equation) is the compressed phase space volume for $r$ after the measurements.

Although we have used the natural log to make the entropy expressions familiar for physicists, in information theory one often uses the binary logarithm, $l b \equiv \log _{2}$. With natural logs the information is in nats, but with $l b$ it is in bits. When expressing information differences in Section 5 we translate to bits. Since a full bit represents a factor of two improvement in the error bar, $\Delta S_{1 \mathrm{f}}(r)$ may only be a fraction of a bit, trivial perhaps, but subtle too, given the mammoth information compression from raw data to this one targeted parameter degree of freedom.

\section{6. $2 \sigma$ Calculation}

We define $\sigma_{95}$ through

$$
\int_{\max \left(0, r_{\mathrm{b}}-\sigma_{95}\right)}^{r_{\mathrm{b}}+\sigma_{95}} \mathcal{L}(r) d r=0.954 \int_{0}^{\infty} \mathcal{L}(r) d r,
$$

where $r_{\mathrm{b}}$ is the best-fit value of $r$. The $\sigma_{95}$-limit is determined by numerically integrating the Gaussian-fitted 1D likelihood curve.

In most cases considered in this paper the likelihood curves turn out to be well approximated by Gaussians. Therefore, when 
there is a low $\sigma$ detection (e.g., for $r=0.12$ ) or when $r \sim 0$, to a very good approximation we have $\sigma_{95}=2 \sigma$ where $\sigma$ is the width of the Gaussian fit. Thus, throughout this paper we will use the common notation of $2 \sigma$, which represents $\sigma_{95}$ and has been calculated through Equation (3). The only exception to this way of determining $2 \sigma$ is when it is being directly given by the inverse of the Fisher matrix, where $\sigma$ represents the width of the likelihood function, under the assumption of its Gaussianity.

\section{CONSTRAINED CORRELATIONS AND LINEAR RESPONSE IN PIXEL-PAIR AND PARAMETER SPACE}

Here we quantify the sensitivity of cosmic parameters to variations in different $\mathcal{C}_{X \ell}$ spectra. We find general forms for filters or "susceptibilities" which relate the linear response of a target variable to the stimulus of a driver variable (with the $\mathcal{C}_{X \ell}$ as a special case) through the CMB data. These filters can also be referred to as window functions to be consistent with the language used for bandpowers, in which the driver is the $\mathcal{C}_{X \ell}$ and the response is the bandpower. The window function attached to each bandpower "gathers" $\ell$-space power for the associated bandpower from the $\mathcal{C}_{X \ell}$ spectrum. There is a long history of making such windows publicly available. They were used in likelihood evaluations in the 2000 release of the BOOMERanG "B98" results (Lange et al. 2001). Tegmark \& de Oliveira-Costa (2001) used similar window functions in a quest for the best quadratic estimator.

In the following we use $\mathbf{q}_{\mathrm{S}}$ for the stimuli (or driver parameters) and $\mathbf{q}_{\mathrm{R}}$ for the response parameters. Assume that $\Delta$ is a realization of the CMB sky for $\mathbf{q}_{\mathrm{R}}=\mathbf{q}_{\mathrm{R}}^{*}$ and $\mathbf{q}_{\mathrm{S}}=\mathbf{q}_{\mathrm{S}}^{*}$. In the attempt to estimate the maximum likelihood parameters from $\Delta$, if $\mathbf{q}_{\mathrm{S}}$ is displaced from its fiducial value by $\delta \mathbf{q}_{\mathrm{S}}$, in response $\mathbf{q}_{\mathrm{R}}$ needs to be readjusted to achieve the (constrained) maximum likelihood. Here we search for this displacement $\delta \mathbf{q}_{\mathrm{R}}$. We Taylor-expand the logarithm of the likelihood function (Equation (2)) to the second order of $\delta \mathbf{q}_{\mathrm{R}}$ around $\mathbf{q}_{\mathrm{R}}=\mathbf{q}_{\mathrm{R}}^{*}$, with the constraint that $\mathbf{q}_{\mathrm{S}}$ is fixed at $\mathbf{q}_{\mathrm{S}}^{*}+\delta \mathbf{q}_{\mathrm{S}}$. We solve for the $\delta \mathbf{q}_{\mathrm{R}}$ that maximizes the likelihood (Bond et al. 1998)

$$
\delta q_{\mathrm{R} \alpha}=\frac{1}{2} \sum_{\beta \in \mathrm{R}}\left[F^{-1}\right]_{\alpha \beta} \operatorname{Tr}\left[\mathbf{C}_{\mathrm{t}}^{-1} \mathbf{C}_{\mathrm{s}, \beta} \mathbf{C}_{\mathrm{t}}^{-1}\left(\left\langle\Delta \Delta^{\mathrm{T}}\right\rangle-\mathbf{C}_{\mathrm{t}}\right)\right],
$$

where $\mathbf{C}_{\mathrm{s}, \beta}=\partial \mathbf{C}_{\mathrm{s}} / \partial q_{\beta}$ and the sum (over $\beta \in \mathrm{R}$ ) only runs over the response parameters. We have approximated the curvature term by its ensemble average, i.e., by the Fisher matrix $\mathbf{F}$,

$$
F_{\alpha \beta} \equiv-\frac{1}{2}\left\langle\frac{\partial^{2} \ln P(\mathbf{q} \mid \Delta, \mathcal{T})}{\partial q_{\alpha} \partial q_{\beta}}\right\rangle=\frac{1}{2} \operatorname{Tr}\left(\mathbf{C}_{\mathrm{t}}^{-1} \mathbf{C}_{\mathrm{s}, \alpha} \mathbf{C}_{\mathrm{t}}^{-1} \mathbf{C}_{\mathrm{s}, \beta}\right),
$$

assuming uniform prior distributions for the parameters. We have also replaced $\Delta \Delta^{T}$ by its ensemble average $\left\langle\Delta \Delta^{T}\right\rangle$ to remove the fluctuations in the response parameters due to cosmic variance. Inserting the first order approximation $\left\langle\Delta \Delta^{T}\right\rangle-\mathbf{C}_{\mathrm{t}}=$ $-\sum_{\mu \in S} \mathbf{C}_{\mathrm{s}, \mu} \delta q_{\mathrm{S} \mu}$ (with the sum $\mu \in \mathrm{S}$ only over stimulus parameters) yields

$$
\delta q_{\mathrm{R} \alpha}=-\sum_{\mu} \delta q_{\mathrm{S} \mu} \sum_{\beta}\left[F^{-1}\right]_{\alpha \beta} F_{\beta \mu}
$$

Note that we have reserved $\alpha$ and $\beta$ for the response parameters and $\mu$ for the stimuli. One can equivalently write

$$
\frac{\delta q_{\mathrm{R} \alpha}}{q_{\mathrm{R} \alpha}}=-\sum_{\mu \in \mathrm{S}} W_{\alpha \mu} \frac{\delta q_{\mathrm{S} \mu}}{q_{\mathrm{S} \mu}}, W_{\alpha \mu}=+\frac{q_{\mathrm{S} \mu}}{q_{\mathrm{R} \alpha}} \sum_{\beta \in \mathrm{R}}\left[F^{-1}\right]_{\alpha \beta} F_{\beta \mu} .
$$

One can consider the $\mathbf{q}_{\mathrm{R}, \mathrm{S}}$ to represent extended parameters of $\mathcal{C}_{X \ell}$ 's as well. A case of special interest for us is when $\mathbf{q}_{\mathrm{S}}$ is the $\mathcal{C}_{X \ell}$ while $\mathbf{q}_{\mathrm{R}}$ is $r$ or another subset of $\mathcal{C}_{X \ell}$ 's. The explicit form of the filter for $\mathbf{q}_{\mathrm{S}}=\mathcal{C}_{X \ell}$ would be

$$
W_{\alpha, X \ell}=+\frac{\mathcal{C}_{X \ell}}{q_{\alpha}} \sum_{\beta}\left[F^{-1}\right]_{\alpha \beta} F_{\beta, X \ell}
$$

\subsection{Linear Response of $C_{B B \ell}$ to $C_{E E \ell}$ : Power Leakage}

We can quantify the leakage of CMB power among different $\mathcal{C}_{X \ell}$ 's, which are already the stimulating drivers in Equation (5), by taking them to be the response variables as well,

$$
W_{X \ell, X^{\prime} \ell^{\prime}}=\frac{\mathcal{C}_{X^{\prime} \ell^{\prime}}}{\mathcal{C}_{X \ell}} \sum_{X^{\prime \prime}, \ell^{\prime \prime}}\left[F^{-1}\right]_{X \ell, X^{\prime \prime} \ell^{\prime \prime}} F_{X^{\prime \prime} \ell^{\prime \prime}, X^{\prime} \ell^{\prime}} .
$$

Here the $X \ell$ and $X^{\prime \prime} \ell^{\prime \prime}$ correspond to the parameters which are being measured, while the $X^{\prime} \ell^{\prime}$ only refer to constrained variables. The case of specific interest in this work is $X=$ $B B, X^{\prime}=E E$, which is the leakage of the larger $E$-mode into $B$-mode. We also consider $X=E E, X^{\prime}=B B$ for comparison. One could investigate the $X=X^{\prime}$ filters, which characterize the mask coupling, but we leave them out as they are not of direct relevance to our power leakage study.

We have verified numerically that for a full sky observation, using the map-based methods gives uncorrelated modes $W_{E E \ell, B B \ell^{\prime}}=W_{B B \ell, E E \ell^{\prime}}=0$. Figure 2 shows the cross filters for an $\ell=100$ stimulus, i.e., $W_{E E \ell, B B 100}$ and $W_{E E \ell, B B 100}$, for $f_{\text {sky }}=0.07$ (at $N_{\text {side }}=64$, pixel size $\approx 56^{\prime}$ ) and $f_{\text {sky }}=0.007$ (at $N_{\text {side }}=128$, pixel size $\approx 28^{\prime}$ ). The observed patches are in the form of spherical caps. (Figure 3 shows the associated beam and pixel window functions along with the polarization power spectra.) We see that the mode correlation increases with decreasing $f_{\text {sky }}$ for a fixed observation time. The high- $\ell$ rise in the filters is due to finite pixel sizes, and hence is more pronounced at lower resolution. We also see that variations in the $E$-mode at most scales have a relatively larger impact on the small $B$ signal compared to the impact of the $B$-mode on the $E$ signal. The width of the oscillation $\Delta \ell \sim \theta_{\text {patch }}^{-1}$ is related to the cap size, narrowing as $f_{\text {sky }}$ goes up. The leakage is larger for smaller $r$, hence must be well characterized for highly sensitive $B$-mode experiments.

\subsection{Linear Response of $r$ to $\mathcal{C}_{B B \ell}$ and $\mathcal{C}_{E E \ell}$}

We now use these filters to quantify the linear response of $r$ to uncertainty in the $\mathcal{C}_{X \ell}$ through the following filter:

$$
W_{r, X \ell}=\frac{F_{r, X \ell}}{F_{r r}} \frac{\mathcal{C}_{X \ell}}{r} .
$$

The filter for a Spider-like experiment with a fiducial $r=$ 0.12 is shown in Figure 4 , as $f_{\text {sky }}$ varies (as does the pixel size). The red, purple, blue, and green curves correspond to $f_{\text {sky }}=0.75,0.25,0.07$, and 0.007 , calculated at $N_{\text {side }}=16$, $N_{\text {side }}=32, N_{\text {side }}=64$, and $N_{\text {side }}=128$ respectively. As expected, the figures show that the measured $r$ is more sensitive to $B B$ than to $E E$ on most scales.

\section{SIMULATION METHODS AND CALCULATIONAL RESULTS}

In this section we use the map-based $T-Q-U$ likelihood procedure of Section 2 to compute the posterior $P\left(\mathbf{q} \mid f_{\text {sky }}, \Delta, \mathcal{T}\right)$ in 

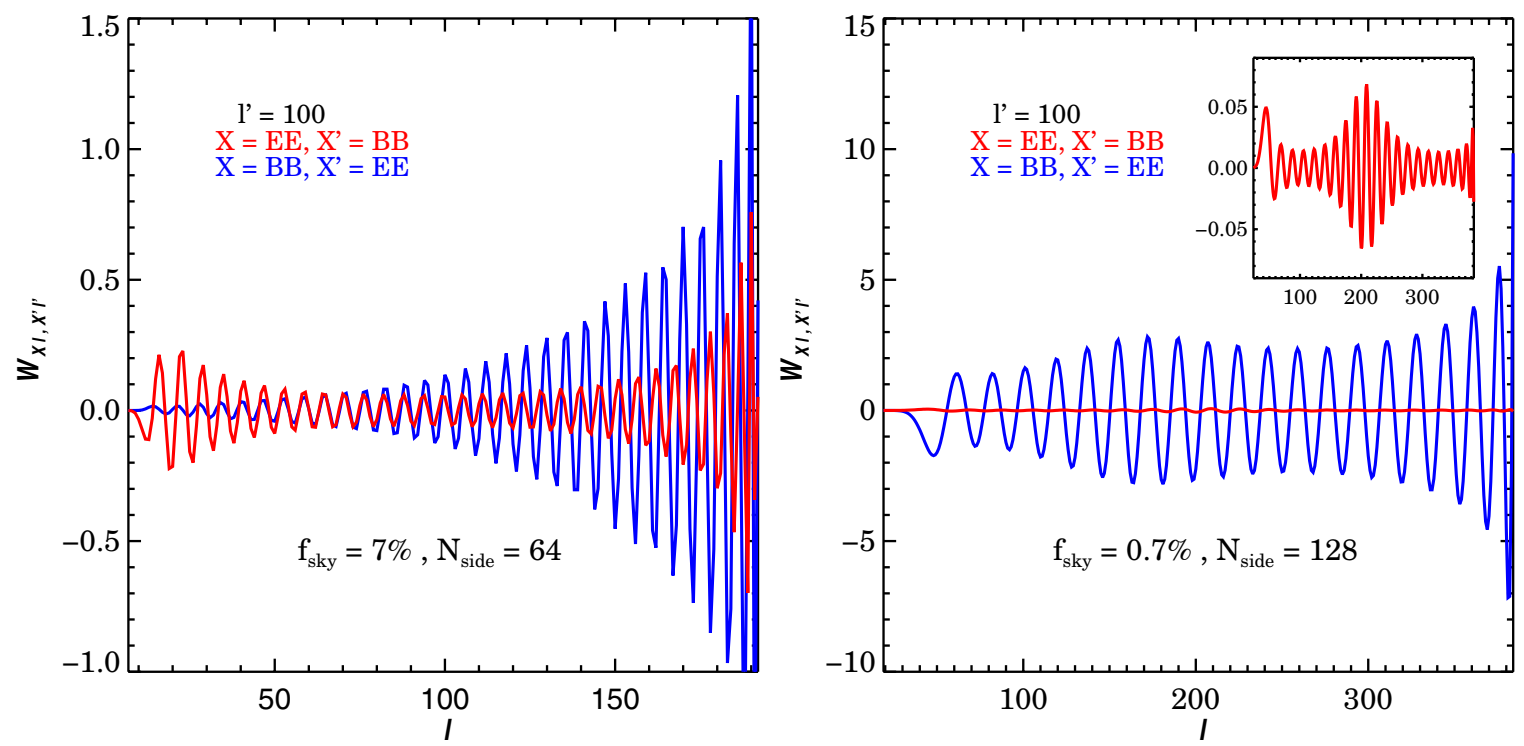

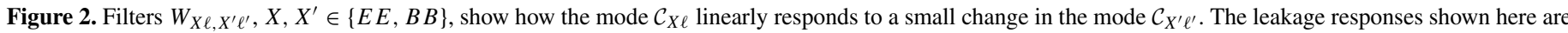

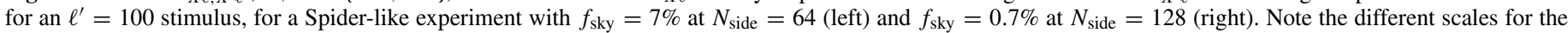
$y$-axis. The corner box of the right panel magnifies $W_{E E \ell, B B 100}$ for $f_{\text {sky }}=0.7 \%$, whose details are not clear in the main plot.

(A color version of this figure is available in the online journal.)

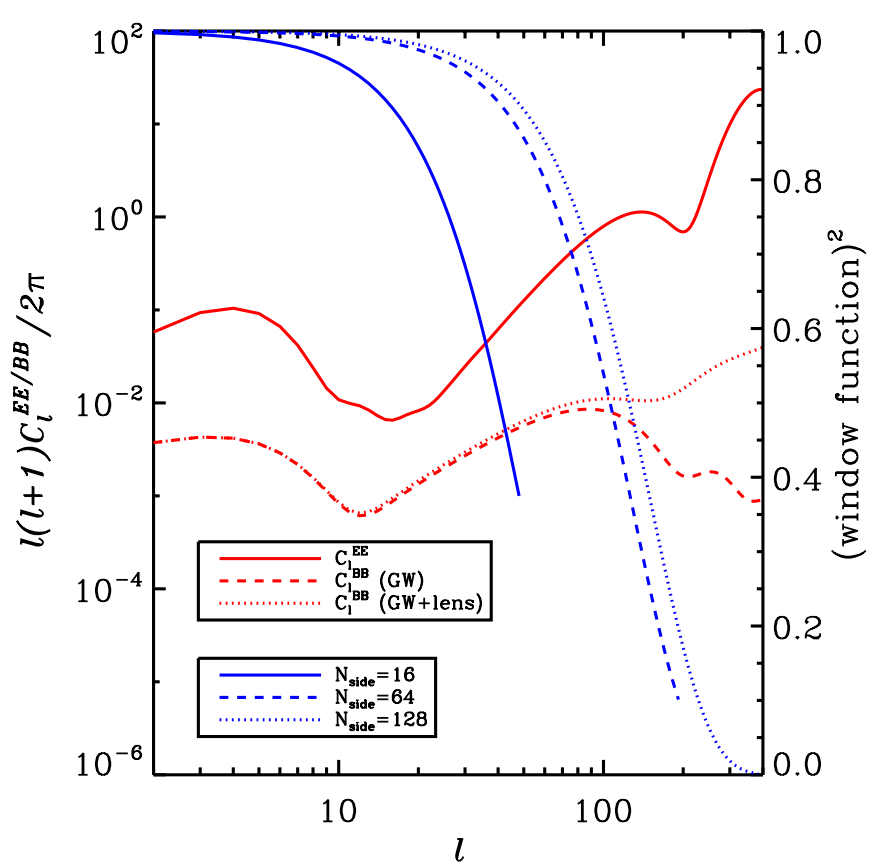

Figure 3. Beam and pixel window functions for different resolutions are compared to the polarization power spectra for the best fit WMAP7-only parameters for the $\Lambda \mathrm{CDM}+$ lensing $+\mathrm{SZ}+$ tensor model, with the addition of a tensor component of strength $r_{\text {fid }}=0.12$. $B$-mode $(\mathrm{GW})$ shows just the gravity wave-induced contribution and $B$-mode ( $\mathrm{GW}+$ lens) includes the lensing contribution as well.

(A color version of this figure is available in the online journal.)

parameter subspaces and, by marginalization, the 1D posterior $P\left(r \mid f_{\text {sky }}, \Delta, \mathcal{T}\right)$ as a function of $f_{\text {sky. }}$. We also make identical calculations to the $T-Q-U$ pixel-based ones in $\ell$-space using $T T, T E, E E$, and $B B$, and assuming no mixing. We show that such a naive approach does quite well in predicting the errors: if properly handled, polarization-mode-mixing is not a significant error source in most cases. Of course for either method to be successful, all generalized noise sources need to be identified including instrumental leakage from $T$ to $Q$ and $U$.

\subsection{Calculation of Ensemble-averaged Posteriors on Parameter Grids}

We calculate the posterior distribution on a gridded parameter space, a method mostly applicable to low dimensional parameter spaces. At each point of the parameter grid the $\mathcal{C}_{X \ell}$ 's are calculated using the public code CAMB. ${ }^{14}$ These are then multiplied by beam windows, $\mathcal{B}_{\ell}^{2}=e^{-\ell(\ell+1) \sigma_{\mathrm{b}}^{2}}$, assuming a Gaussian beam of width $\sigma_{\mathrm{b}}=0.425 \theta_{\mathrm{FWHM}}$, and by pixelization windows $W_{\mathrm{pix}, \ell}^{2}$, an isotropized approximation to finite pixel size effects. (Timestream digitization filters are also generally required, but are swamped by these two filters.) The product is used to construct the symmetric $3 N_{\text {pix }} \times 3 N_{\text {pix }}$ theoretical pixel-pixel signal covariance matrices, with six independent sub-matrices, $\mathbf{C}_{\mathrm{s}, X}, X \in\{T T, T Q, T U, Q Q, Q U, U U\}$. We assume that experimental noise is Gaussian and usually take it to be white. Therefore, $\mathbf{C}_{\mathrm{n}, T}=\sigma_{\mathrm{n}, \mathrm{T}}^{2}$ I for the temperature block and $\mathbf{C}_{\mathrm{n}, Q, U}=\sigma_{\mathrm{n}, \mathrm{pol}}^{2} \mathbf{I}$ for the polarization block of the covariance matrix, where we usually have $\sigma_{\mathrm{n} \text {,pol }} \sim \sqrt{2} \sigma_{\mathrm{n}, \mathrm{T}}$. Here the $\sigma_{n}$ 's are effective noises per pixel, an amalgamation of the noises coming from different frequency channels. I is the identity matrix. We neglect leakage from $T$ to $Q$ and $U$.

Since we are forecasting the uncertainties in $r$ from different experimental setups, and not analyzing actual CMB maps, we can bypass creating a large ensemble of simulated CMB maps by replacing the observed correlation matrix $\mathbf{C}_{\mathrm{t}, \mathrm{O}} \equiv \Delta \Delta^{T}$ by its ensemble average:

$$
\operatorname{Tr}\left(\mathbf{C}_{\mathrm{t}}^{-1}(\mathbf{q}) \mathbf{C}_{\mathrm{t}, \mathrm{O}}\right) \rightarrow \operatorname{Tr}\left(\mathbf{C}_{\mathrm{t}}^{-1}(\mathbf{q}) \overline{\mathbf{C}}_{\mathrm{t}, \mathrm{O}}\right)
$$

Here $\overline{\mathbf{C}}_{t, \mathrm{O}}$ is the ensemble-averaged "pixel-pair data," namely the covariance matrix of the input fiducial signal model together with the instrument noise, and $\mathbf{C}_{t}(\mathbf{q})$ is the signal pixel-pixel

\footnotetext{
14 http://camb.info/
} 


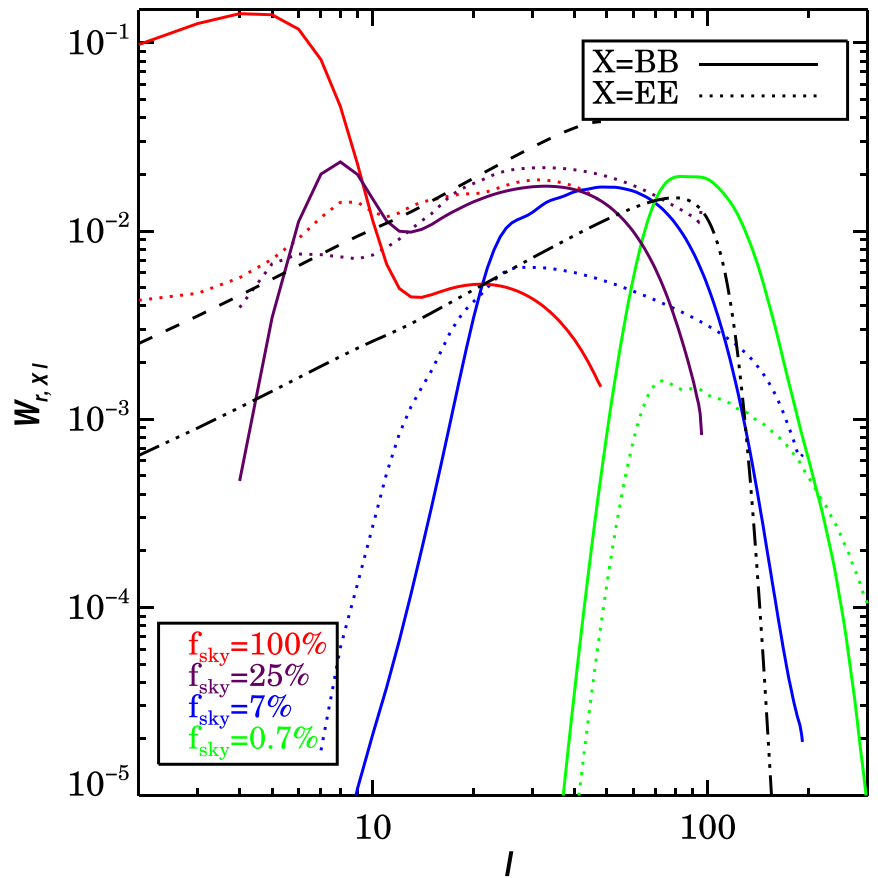

Figure 4. Window functions $W_{r, X \ell}$ for $X \in\{E E, B B\}$ for different sky cuts show that, as expected, all-sky experiments are nicely sensitive to the reionization $B B$ bump, but smaller sky experiments are not, although they pick up well the $\ell \sim 50-100$ region. We have used $r=0.12$ for the fiducial model. The rapid declines to high $\ell$ are more due to the onset of experimental noise than to the onset of the lensing-induced $B$ "noise." Residual foreground noise has not been included in these plots. Note that even a coverage with $f_{\text {sky }}$ only 0.007 can punch out a robust detection from 50 to 150 in $\ell$. The coverage with $f_{\text {sky }}=0.07$, although it loses out a bit (relatively) at 150 , its detection would come from a wider stretch in $\ln \ell$, out to $\ell \sim 20$ before falling off. Only at $f_{\text {sky }}>0.25$ does one begin to pick up the reionization bump. The curious drop in the all-sky $N_{\text {side }}=16$ red line at the top is due to the Spider-like noise for higher $\ell$ being heavily enhanced because all of the sky is covered in the same amount of observing time. To illustrate the role of this, a CMBPol-like experiment with $C_{\mathrm{N}}$ decreased by $\sim 1000$ is plotted, with $N_{\text {side }}=16$ (dashed straight line) and $N_{\text {side }}=64$ (triple-dot-dashed line). The reason all three are offset from one another is because the normalizing $\sigma_{r}^{2}$ depends upon the amount the filter captures of the total $r$ signal.

(A color version of this figure is available in the online journal.)

covariance matrix for the parameters $\mathbf{q}$ plus the various noise contributions, instrumental and otherwise. An advantage of this approach is that the recovered values of the parameters are what the ensemble average of sky realizations would yield and will not move hugely due to the chance strangeness of any one realization (as the real sky may provide for us). Note that while sample variance does not impact the location of the maximum likelihood in this ensemble-averaged approach, it is fully reflected in the width of the posterior distribution from which our uncertainties are derived.

We mask out the part of Galaxy falling in the observed patch (the P06 WMAP-mask in Page et al. 2007), assuming it to be too foreground-dominated for useful parameter extraction. We also project out modes larger than the fundamental mode of the observed patch since, due to time-domain filtering, information is not usually recoverable on such large scales. For instance, if the mask has the shape of a spherical cap extending from the north pole to $\theta=\theta_{\text {patch }}$, we add a very large noise to the modes with $2 \ell+1<[2 \pi / \varpi]$ where [...] takes the integer part and $\varpi=2 \sin \left(\theta_{\text {patch }} / 2\right)$ is the flat $2 \mathrm{D}$ radius of the disk with an area equal to the solid angle of the cap. This makes the likelihood insensitive to any information at and beyond the patch scale. This large scale mode cut is especially important to include for larger values of $f_{\text {sky }}$, where the low $\ell$ modes contribute significantly to $r$ measurement through the reionization bump. In real large sky experiments it will not be easy to draw such modes from maps.

Our simulations cover two observational cases: an all-sky experiment with Planck-like white noise levels, and a partial sky experiment with Spider-like white noise levels, each with two frequency channels, assuming other frequencies are used for subtracting foregrounds. We have also made the simplifying assumption that in each experiment, the FWHM of both channels is the same as the channel with the larger beam. This does not affect the results much due to the crude size of the pixelization and the absence of the gravitational wave signal at small scales. See Table 1 for other experimental assumptions.

For the Spider-like case we keep the flight time constant so that the observation gets deeper as $f_{\text {sky }}$ decreases, while for the Planck-like experiment the pixel noise is assumed constant for different values of $f_{\text {sky }}$. The latter case, with small values of $f_{\text {sky }}$, is used to illustrate how well a strategy of only analyzing the lowest foreground sky could work, if, e.g., foreground removal turns out to be prohibitive over much of the sky. If foregrounds can be well removed from Planck, then full sky is appropriate.

We calculate the constraints on targeted cosmological parameters for different $f_{\text {sky }}$ 's, assuming the observed patches are spherical caps from $\theta=0$ to $\theta=\theta_{\text {patch }}$, corresponding to $\theta=\cos ^{-1}\left(1-2 f_{\text {sky }}\right)$. We perform the analysis at different resolutions for different sky cuts to minimize the effect of pixelization for small $f_{\text {sky }}$ on the one hand, and to keep the computational time reasonable for large $f_{\text {sky }}$ on the other hand. We use $N_{\text {side }}=32, N_{\text {side }}=64$, and $N_{\text {side }}=128$ for $f_{\text {sky }}>0.25,0.007<f_{\text {sky }} \leqslant 0.25$, and $f_{\text {sky }} \leqslant 0.007$, respectively. We checked the results for two neighbor resolutions at resolution switches. For the low $f_{\text {sky }}$ switch, the results are not sensitive to the change of resolution while for the switch at larger $f_{\text {sky }}$ we are about $10 \%-15 \%$ pessimistic in the results by choosing the lower resolution, specifically for a Planck-like case (with small beam) and for a higher value of $r$, e.g., $r=0.12$. In these cases, lensing starts to dominate at higher multipoles and choosing a high enough resolution for the analysis would improve the errors on $r$ by resolving the primordial gravity waves at relatively high multipoles.

\subsection{Residual Foreground-subtraction "Noise"}

No study of gravitational wave detectability by $B$-mode experiments can ignore the impact of polarized foreground emission. Component separation is a major industry in itself. Various techniques have been utilized with CMB data up to now which often involve template parameter marginalization of one sort or another (see, e.g., Efstathiou et al. 2009 and references therein). We have been lucky so far in that the foregrounds have been manageable for $T T, T E$, and $E E$. The level of subtraction needed to unearth the very tiny gravity wave-induced $B$-signal is rather daunting, especially since the foregrounds are largest at the low $\ell$. Thus, although we may wrestle the generalized noise from the detectors and from experimental systematics to levels allowing small $r$ to be detectable, the foregrounds will need to be well addressed before any claim of primordial detection will be believable. Although we have learned much already about the $T T$ foregrounds and, from WMAP, the synchrotron $E E$ (Gold et al. 2011), we do not know the $\ell$-shape or the amplitude of the polarization for dust.

In O'Dea et al. (2011, 2012), the polarization emission from thermal dust is based on a three-dimensional model of 
Table 1

Specifications of Spider-like, Planck-like and CMBPol (Mid-cost) Experiments for Simulations

\begin{tabular}{|c|c|c|c|c|c|c|}
\hline Experiment & $\begin{array}{c}\text { Frequency } \\
(\mathrm{GHz})\end{array}$ & FWHM & Num. of Det. & $\begin{array}{c}\Delta T^{\mathrm{a}} \\
I\end{array}$ & $\begin{array}{c}\Delta T \\
Q \& U\end{array}$ & Obs. Time \\
\hline Spider-like $^{b}$ & 96 & $50^{\prime}$ & 768 & 3.2 & 4.5 & $580 \mathrm{hr}$ \\
\hline Spider-like & 150 & $32^{\prime}$ & 960 & 2.7 & 3.8 & $580 \mathrm{hr}$ \\
\hline Planck-like ${ }^{\mathrm{c}}$ & 100 & $10^{\prime}$ & 8 & 3.8 & 6.1 & $2.5 \mathrm{yr}$ \\
\hline Planck-like & 143 & $7^{\prime}$ & 8 & 2.4 & 4.6 & $2.5 \mathrm{yr}$ \\
\hline$C M B P o l(\text { mid-cost })^{\mathrm{d}}$ & 100 & $8^{\prime}$ & $\ldots$ & 0.18 & 0.26 & $\ldots$ \\
\hline CMBPol (mid-cost) & 150 & $5^{\prime}$ & $\ldots$ & 0.19 & 0.27 & $\ldots$ \\
\hline
\end{tabular}

Notes.

${ }^{a} \mathrm{nK}$, the instrument sensitivity divided by $\sqrt{\text { total observation time }}$.

b These Spider-like specifications which are used as the default in this paper are different from the ones proposed in Fraisse et al. (2013) with two 20 day flights. The first flight uses three 90 and three $150 \mathrm{GHz}$ receivers each with 288 and 512 detectors respectively. In the second flight, two $280 \mathrm{GHz}$ receivers replace one 90 and one $150 \mathrm{GHz}$ telescope, leaving the configuration of the flight identical to the first one. The detector sensitivity as proposed in Fraisse et al. (2013) is 150, 150 and $380 \mu \mathrm{K}_{\mathrm{CMB}} \sqrt{\mathrm{s}}$ at 90,150 and $280 \mathrm{GHz}$, respectively. The performance of the default Spider-like experiment in this paper and the more recent proposal as in Fraisse et al. (2013) are very close (see Figure 1).

${ }^{\mathrm{c}}$ http://www.rssd.esa.int/index.php?project=planck.

${ }^{\mathrm{d}}$ For a mid-cost full-sky CMBPol experiment based on Table 13 of Baumann et al. (2009). We are using 100 and 150 $\mathrm{GHz}$ channels in our simulations. Adding more channels, in the unrealistic case of no foreground contamination that we simulate, would not affect the limits on $r$ since with these low instrument noise levels, either lensing or cosmic variance, depending on how small $r$ is, would be the dominant source of uncertainty.

dust density and two-component Galactic magnetic field. It is assumed that the degree of polarization has a quadratic dependence on the magnetic filed strength and its direction is perpendicular to the component of the local magnetic field in the plane of the sky, similar to the model assumed by WMAP in Page et al. (2007). In forecasting for proposed post-Planck satellite experiments, simple approximations for thermal dust and synchrotron emission have been made (e.g., Baumann et al. 2009 , and references therein). The dusty $\ell$-structure in this model is similar to the O'Dea et al. (2011) form: $\mathcal{C}_{X \ell} \sim \ell^{-0.5}$ for $X=E E, B B$. We follow the Baumann et al. (2009) approach here, but apply it to our pixel-based analysis.

We therefore assume that the maps are already foregroundsubtracted, possibly with the wider Planck frequency coverage used in conjunction with the Spider maps, with the CMBcomponent having a residual uncertainty, which we incorporate in our analysis as an additional large-scale (inhomogeneous) noise component $C_{\mathrm{N}}^{(\mathrm{fg})}$. We assume the power spectrum of the foreground residuals has the same shape as the original foreground spectrum, but with only a few percent of the amplitude

$$
\mathcal{C}_{X \ell} \rightarrow \mathcal{C}_{X \ell}+\sum_{\mathrm{fg}=\mathrm{S}, \mathrm{D}} \epsilon_{X}^{(\mathrm{fg})} \mathcal{C}_{X \ell}^{(\mathrm{fg})}, X=E E, B B,
$$

with the sum over synchrotron (S) and dust (D) emissions. The tunable removal-efficiency parameters $\epsilon^{(\mathrm{fg})}$ are taken to be $5 \%$ in our plots. The shapes are

$$
\begin{aligned}
\mathcal{C}_{X \ell}^{(\mathrm{S})}(v)= & \frac{\ell(\ell+1)}{2 \pi} A_{\mathrm{S}}\left(\frac{v}{v_{0}}\right)^{2 \alpha_{\mathrm{S}}}\left(\frac{\ell}{\ell_{0}}\right)^{\beta_{\mathrm{S}}} \\
\mathcal{C}_{X \ell}^{(\mathrm{D})}(v)= & \frac{\ell(\ell+1)}{2 \pi} p^{2} A_{\mathrm{D}}\left(\frac{v}{v_{0}}\right)^{2 \alpha_{\mathrm{D}}}\left(\frac{\ell}{\ell_{0}}\right)^{\beta_{\mathrm{D}}} \\
& \times\left[\frac{e^{h v_{0} / k T}-1}{e^{h v / k T}-1}\right]^{2} .
\end{aligned}
$$

The dust polarization fraction, $p$, is assumed to be around $5 \%$. The values for the other parameters taken from Baumann et al.
Table 2

Parameters of our Assumed Foreground Model, Adopted from Baumann et al. (2009)

\begin{tabular}{lcr}
\hline \hline Parameters & Synchrotron & Dust \\
\hline$A_{S, D}\left(\mu K^{2}\right)$ & $4.7 \times 10^{-5}$ & 1 \\
$\nu_{0}$ & 30 & 94 \\
$\ell_{0}$ & 350 & 10 \\
$\alpha$ & -3 & 2.2 \\
$\beta^{E}$ & -2.6 & -2.5 \\
$\beta^{B}$ & -2.6 & -2.5 \\
\hline
\end{tabular}

(2009) are listed in Table 2. They were chosen to give agreement with WMAP, DASI and IRAS observations (and the Planck sky model, which is based on these). Although this model provides only a rough guide to the impact that incomplete foreground subtraction will have on $r$-estimation, it does include the crucial large-scale dependence which differentiates it so much from the structure of the instrumental noise.

A natural question when considering deep small sky observations is how many patches there are on the sky with low foregrounds so the requisite cleaning is at a minimum. The Planck Sky Model for the polarized foreground emission (Leach et al. 2008; Delabrouille et al. 2012) is similar to the one we have adopted. Using a code developed by Miville-Deschênes, we have calculated for patches of radius $R$ the pixel-averaged variance at pixel $p, \sigma_{\text {pol,fg }}^{2}(p, R)=\left\langle(P-\bar{P}(<R))^{2}\right\rangle$ of the polarization intensity $P=\sqrt{Q^{2}+U^{2}}$ about the patch-average $\bar{P}$ arising from the synchrotron and dust foregrounds. We compare this with the $\sigma_{\mathrm{pol}, \mathrm{gw}}^{2}(p, R)$ we obtain for each patch in a single tensor-only primordial polarization realization (which is proportional to $r^{2}$ ). The patches are sorted in decreasing order of the "signal-to-noise" ratio $\sigma_{\mathrm{pol}, \mathrm{gw}}(p, R) / \sigma_{\mathrm{pol}, \mathrm{fg}}(p, R)$. The next pixel on the list is included in a patch list if it has no overlap with the patches in the previously determined higher signal-to-noise list. A patch is considered to be $r$-clean if this polarization signalto-noise exceeds unity, a rather strong criterion. At $100 \mathrm{GHz}$, we found no " $r=0.01$ "-clean patches, 7 " $r=0.05$ "-clean patches 
and 10 " $r=0.1$ "-clean patches with $f_{\text {sky }} \gtrsim 0.007\left(R=10^{\circ}\right)$. There are one " $r=0.05$ "-clean patch and two " $r=0.1$ "-clean patches for $f_{\text {sky }} \gtrsim 0.03\left(R=20^{\circ}\right)$. At $150 \mathrm{GHz}$, we found no " $r=0.05$ "-clean patches and one " $r=0.1$ "-clean patch with $f_{\text {sky }} \gtrsim 0.007$ but no $r=0.1$-clean patches for $f_{\text {sky }} \gtrsim 0.03$.

The non-overlapping criterion is quite severe. Another measure of $r$-cleanliness is to determine the fraction of sky with $\sigma_{\text {pol,gw }}(p, R) / \sigma_{\text {pol,fg }}(p, R)$ above unity. The $r$-clean fraction is clearly $\sim 0$ for those values of $r$ and $R$ with no corresponding clean patches (as stated above). Here only the non-zero values are reported. At $100 \mathrm{GHz}$, the " $r=0.05$ "-clean fraction is $\sim 0.14\left(R=10^{\circ}\right)$ and the " $r=0.1$ "-clean fraction is $\sim 0.24$ ( $R=10^{\circ}$ ). For both values of $r$, there is no appreciable decrease in the sky fraction by increasing the patch sizes to $R=20^{\circ}$. At $150 \mathrm{GHz}$, the " $r=0.1$ "-clean fraction is $\sim 0.04\left(R=10^{\circ}\right)$. It should be noted that as these sky fractions do not necessarily correspond to contiguous regions, the sky fraction of interest for small-sky $B$-mode experiments is in principle smaller. The Planck sky model at the lower frequencies agrees with the (extrapolated) synchrotron emission from WMAP, but the higher frequency polarized dust emission really requires the better observations of the Planck mission.

\subsection{Correlations of $r$ with Other Cosmic Parameters}

Either detecting $r$ or placing a tight upper bound is crucial for progress in inflation studies. Correlations of $r$ with other parameters $q_{\alpha}$ must be properly accounted for, since they are marginalized in the reduction to the $1 \mathrm{D} r$-posterior. The relative importance of the various $q_{\alpha}$ is determined by calculating the posterior-averaged cross-correlations $\rho_{r \alpha} \equiv\left\langle\delta r \delta q_{\alpha}\right\rangle_{\mathrm{f}}$, which depend upon the experimental configuration and its noise. Within the Gaussian approximation for the posterior distribution, the correlations can be estimated from the inverse components, $\left[F^{-1}\right]_{r \alpha}$, using the Fisher matrix equation (Equation (4)). Lensing and instrumental noise are included in the generalized noise matrix. Here $F$ is determined from numerical differentiation by taking small steps in the main parameters of the standard $\Lambda$ CDM model $\left(\ln \left(\Omega_{\mathrm{b}} h^{2}\right), \ln \left(\Omega_{\mathrm{c}} h^{2}\right), H_{0}, n_{\mathrm{s}}, \tau, r\right)$ from the fiducial WMAP7 values. ${ }^{15}$ The scalar amplitude $A_{\mathrm{s}}$ is treated as a normalization parameter here, so it is not included in the parameter list. We use two different fiducial values for $r, 0.2$ and 0.01, and three values of $f_{\text {sky }}, 0.007,0.07$, and 0.75 , for a Spider-like experiment. We use a Gaussian prior on all parameters $q_{\alpha}$ but $r$, with the mean and width given by the WMAP7 measurements. For these parameters we choose $\left(F_{\text {prior }}\right)_{\alpha \beta}=\sigma_{\alpha \text {,WMAP7 }}^{-2} \delta_{\alpha \beta}$, which gives a weaker prior than the true WMAP7 results would give. In the quadratic approximation to the posterior information action, the correlation of $r$ with other parameters is approximated by

$$
\rho_{r \alpha} \approx \frac{\left[F_{\mathrm{t}}^{-1}\right]_{r \alpha}}{\sqrt{\left[F_{\mathrm{t}}^{-1}\right]_{r r}\left[F_{\mathrm{t}}^{-1}\right]_{\alpha \alpha}}},
$$

where $F_{\mathrm{t}}=F+F_{\text {prior }}$ is the total Fisher matrix.

For the full sky case, we find the largest $\rho_{r \alpha}$ for $\tau$ and $n_{\mathrm{s}}$, with $\rho_{r \tau}$ and $\rho_{r n_{\mathrm{s}}}$ both $\approx 0.25$. For smaller sky coverage, the degeneracy between $r$ and $\tau$ disappears since the main constraints on $\tau$ come from the large scale polarization, which small cut-sky cases are not sensitive to. The dominant correlations of $r$ are with

\footnotetext{
$\overline{15}$ http://lambda.gsfc.nasa.gov/product/map/dr4/params/lcdm_sz_lens_ wmap7.cfm
}

Table 3

$\sigma_{r}$ from the Full Likelihood Computed on a 2D $r-\tau$ Grid (Bottom), cf. 1D, 2D and 6D Fisher Determinations $\left[F^{-1}\right]^{r r}$, Using Pixel-space Matrices (Middle) and the Simplified $\ell$-space Sums, with $r_{\text {fid }}=0.12$

\begin{tabular}{lcccc}
\hline \hline Method & Parameter Space & $\begin{array}{c}N_{\text {side }}=32 \\
f_{\text {sky }}=1\end{array}$ & $\begin{array}{c}N_{\text {side }}=64 \\
f_{\text {sky }}=0.07\end{array}$ & $\begin{array}{c}N_{\text {side }}=128 \\
f_{\text {sky }}=0.007\end{array}$ \\
\hline Fisher & 1 parameter & 0.022 & 0.018 & 0.037 \\
$\ell$-space & 2 parameter & 0.023 & 0.018 & 0.037 \\
& 6 parameter & 0.025 & 0.020 & 0.037 \\
\hline Fisher & 1 parameter & 0.022 & 0.019 & 0.034 \\
pixel-space & 2 parameter & 0.023 & 0.019 & 0.034 \\
& 6 parameter & 0.025 & 0.020 & 0.035 \\
\hline grid-based & 2 parameter & 0.021 & 0.018 & 0.036 \\
\hline
\end{tabular}

Note. This demonstrates that the use of reduced parameter spaces gives robust results, independent of cap sizes, here for $f_{\text {sky }}=1,0.07,0.007$.

the matter density parameters $\Omega_{\mathrm{c}} h^{2}$ and $\Omega_{\mathrm{b}} h^{2}$, at the $0.1-0.2$ level, a consequence of the gravitational lensing-induced $B B$ noise. Note that under the quadratic approximation the conditional uncertainty in $r$ for given $\bar{q}_{\alpha}$ is $\left\langle\delta r^{2} \mid \bar{q}^{\alpha}\right\rangle=\sigma_{r}^{2}\left(1-\rho_{r \alpha}^{2}\right)$, where $\sigma_{r}$ is the fully marginalized error on $r$. As a result, even in the $25 \%$ case for $\rho_{r \alpha}$, the error diminishes only by $3 \%$ for fixed $q_{\alpha}$ relative to the full $\sigma_{r}$.

Thus we should be able to safely estimate the error on $r$ with all or none of the basic cosmic parameters held fixed. We verified this explicitly by comparing the 2D uncertainties calculated from the full 2D $r-\tau$-grid with the full $6 \mathrm{D}$ uncertainties calculated from the inverse Fisher matrix, in $\ell$-space and in pixel space, in Table 3, for different $f_{\text {sky }}$ and at different resolutions, defined here by the value of $N_{\text {side }}$. With all six parameters included, $\sigma_{r}$ increases by only $\sim 10 \%$ over the single $\tau$-marginalized $\sigma_{r}$, which justifies our exploration using a heavily truncated parameter space to determine the errors on $r$.

\subsection{Results in $r-\tau$ Space}

In this section, we use $\tau$ as well as $r$ to make our 2D parameter space since it has a direct impact on the $B B$ reionization bump. We fix the overall $\mathcal{C}_{\ell}$ normalization for each parameter pair to the WMAP TT measurement at $\ell=220$. This is equivalent to having $A_{\mathrm{s}}$ as an adjustable parameter. If not otherwise stated, lensing has been included in all of the following simulations with a fixed noise template, linearly scaled with $A_{\mathrm{s}}$ accordingly. Treating lensing in the noise covariance completely takes into account its effect on sample variance. It may be possible for it to be partly removed in the patch using delensing algorithms (see, e.g., Smith et al. 2008 and references therein), leading to a reduced variance in the same way that we are treating a foreground residual. However, treating lensing as a noise source is a good assumption for our purposes here.

The $2 \sigma_{r}\left(f_{\text {sky }}\right)$ plots in Figures 5 and 6 are our main results. Shown are two fiducial models with $r_{\text {fid }}=0.12,0.001$, both having $\tau_{\text {fid }}=0.09$. The $f_{\text {sky }}$ in the plots is the sky coverage before the Galaxy is masked. The Galaxy cut starts coming into the observed patch for $\theta_{\text {patch }} \sim 40^{\circ}$.

The results are compared to the expected error bars on $r$ from a simplified $\ell$-space analysis. Proper mode counting is a difficulty in the $\ell$-space approximation for cut-skies. (This differs from the full pixel-pixel covariance matrix analysis in which all modes are naturally taken care of.) For the $\ell$-space approximation, we have taken the mode number to be the naive 

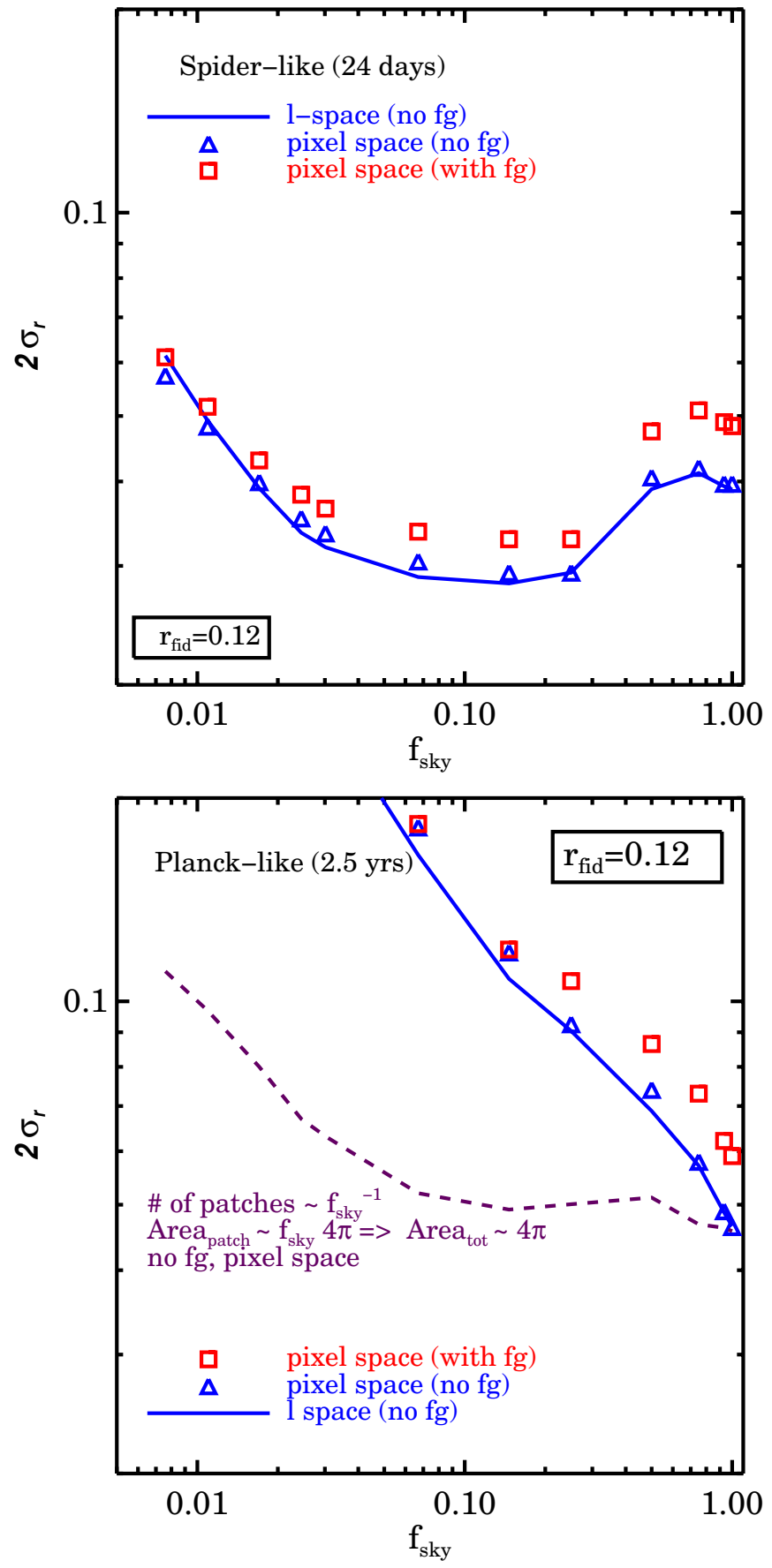

Figure 5. Uncertainty in measuring $r$ for different sky coverages with Spiderlike (top) and Planck-like (bottom) experiments, with and without foregrounds (squares and triangles respectively), for the fiducial model $r_{\text {fid }}=0.12$. The solid lines are the results of $\ell$-space analysis (ignoring foregrounds). The analysis has been performed with different resolutions for different $f_{\text {sky }}$, ranging from $N_{\text {side }}=32$ for full sky to $N_{\text {side }}=128$ for the smallest sky coverage. The $f_{\text {sky }}$ refers to the sky coverage before applying the Galactic cut so for full sky $f_{\text {sky }}$ is effectively $\sim 0.75$. The dashed line is the $2 \sigma_{r}$ if the full sky needs to be effectively considered as a combination of several smaller patches with the individual observed sky fraction being $f_{\text {sky }}$ and the total area of all patches equal to the Galaxy-masked full sky.

(A color version of this figure is available in the online journal.)

$\left[f_{\text {sky }}(2 \ell+1)\right]$ where $[\cdots]$ indicates the integer part. This imposes a low $\ell$-cut on the modes by demanding $\left[f_{\text {sky }}(2 \ell+1)\right] \geqslant 1$ which overrides the $\ell$-cut from the fundamental mode of the patch, $2 \ell+1=\left[2 \pi / 2 \sin \left(\theta_{\text {patch }} / 2\right)\right]$, up to $\theta \approx 30^{\circ}$.
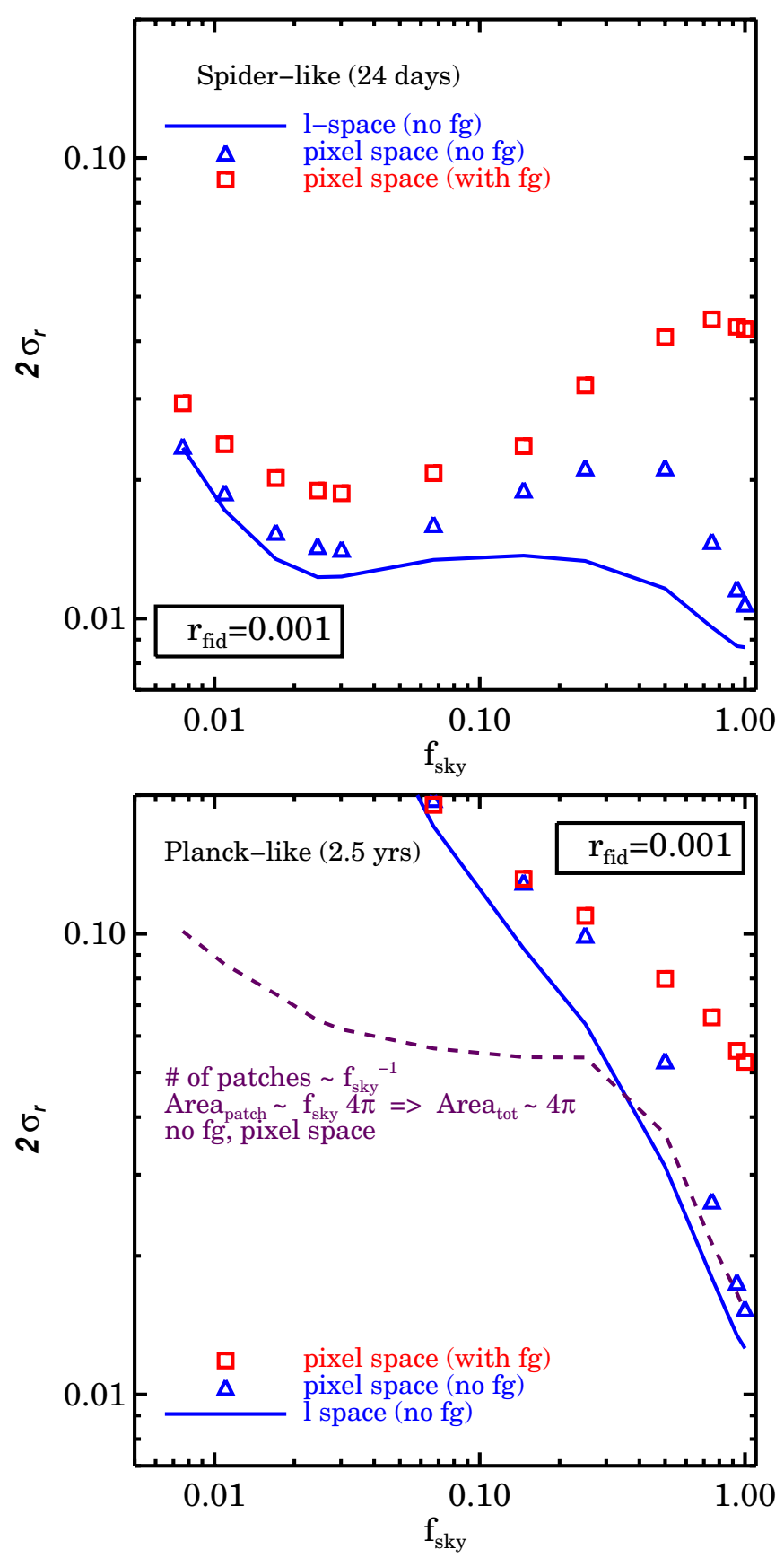

Figure 6. Similar to Figure 5 with $r_{\text {fid }}=0.001$.

(A color version of this figure is available in the online journal.)

This $\ell$-space $\sigma_{r}\left(f_{\text {sky }}\right)$ is a lower bound since it ignores the mode mixing on the cut sky. Still, in the absence of systematic errors and for the simplified noise assumed here, the errors we find are near the true (matrix) values, as Figure 5 confirms for $r_{\text {fid }}=0.12$. A similar measurement with $r_{\text {fid }}=0.2$ shows the same thing, though with a more-flattened curve for $\sigma_{r}\left(f_{\text {sky }}\right)$ for the Spider-like case and with foregrounds playing a smaller role. $E-B$ mixing does not seem to be a serious impediment, at least down to $f_{\text {sky }} \approx 0.01$. For the Spider-like experiment, the error minimum is $2 \sigma_{r}=0.035$ for $r_{\text {fid }}=0.12$, at $f_{\text {sky }} \approx 0.15$, but the trough is broad. For the low $r_{\text {fid }}=0.001$, for which only an upper limit can be expected, Figure 6 shows the agreement in $\sigma_{r}\left(f_{\text {sky }}\right)$ between $\ell$-space and pixel-space is not quite as good. This is especially true for $f_{\text {sky }} \approx 0.25-0.5$ for which 
considerable observation time is expended on the $\ell \approx 12 \mathrm{BB}$ valley (see Figure 3 ) where there is little signal. The naive $\ell-$ space approximation underestimates this, but agreement with pixel-space is regained in runs with the reionization bump removed, by setting $\tau=0$; for this case the monotonic rise in $\sigma_{r}\left(f_{\text {sky }}\right)$ with increasing $f_{\text {sky }}$ continues to full sky.

Extending to the full Galaxy-masked sky improves the upper limit on $r$ since the window function captures the low- $\ell$ bump. The $\ell$-space and pixel-space calculations disagree slightly, but when the Galaxy mask is removed, the estimates agree.

At small $f_{\text {sky }}, 2 \sigma_{r}$ increases due to lensing which dominates the total $B B$ spectrum at small scales. The competition between avoiding contamination by lensing and avoiding the $\ell \approx 12$ valley produces a weak minimum in $\sigma_{r}$ at $f_{\text {sky }} \approx 0.15$ for $r=0.12$, when a detection is expected, and at $f_{\text {sky }} \approx 0.03$ for $r=0.001$, when an upper limit is expected. The full sky is weakly optimal for setting an upper limit in the absence of foregrounds.

The Planck-like measurements in the lower plots of Figures 5 and 6 show a rise in $2 \sigma_{r}$ as $f_{\text {sky }}$ drops. In this case, the information on the large scales are lost while the pixel noise stays unchanged. The dashed lines in these plots show the approximate $2 \sigma_{r}$ for a full-sky Galaxy-masked Planck-like experiment if the large-scale modes are filtered, e.g., by timedomain filtering or due to high foreground contamination and thus the observed region is considered to be a combination of smaller patches (adding up to the full sky in total observed area).

Not surprisingly, we see that foregrounds mostly affect experiments with larger $f_{\text {sky }}$, and for fiducial models with smaller $r$. We also see that deep observations of quite small patches seem to do as well as larger patches (observed less deeply) and even much better if $r$ is small (for which the sample variance is very small and instrument noise plays the dominant role).

Figure 7 shows how different components contribute to the error on $r$ calculated using the Fisher matrix for various $r_{\text {fid }}$ and $f_{\text {sky }}=0.007$ and 0.07 . As before the mode mixing is ignored in the $\ell$-space calculation. If there were no lensing and no mode-mixing, in the limit of no instrument noise, the only source of error would be the sample variance, which is, as expected, proportional to $r$. The solid black lines show the minimum irreducible errors due to sample variance and lensing. We contrast this with calculations in both pixel and $\ell$-space of two Spider-like experiments. One has 10 times less noise than the fiducial Spider case. This noise level can be seen to give almost no contribution to the errors for these sky cuts since lensing noise is dominant. The other has our standard Spider-like noise, which can be seen to significantly add to the error. The impact of neglecting mode mixing in determining $\sigma_{r}$ vanishes as $r$ increases, since sample variance dominates the error, as a comparison of the curves from the pixel-space and $\ell$-space analyses shows. The overplotted symbols represent the errors from measuring the likelihood curve in a gridded $2 \mathrm{D}$ parameter space (as explained earlier). The $2 \sigma_{r}$ 's from the full method and the Fisher matrix approximation are close. The small difference is because the $r$-likelihood curve is not a perfect Gaussian.

Figure 8 shows the 2D $r-\tau$ contours for three different values of sky coverage for a Spider-like experiment compared to a full-sky Planck-like experiment (with Galaxy mask cut), with and without foreground contamination. As expected, $\tau$ is unconstrained as $f_{\text {sky }}$ decreases for the Spider-like experiment since $\tau$-constraints come from the largest angular scales: what
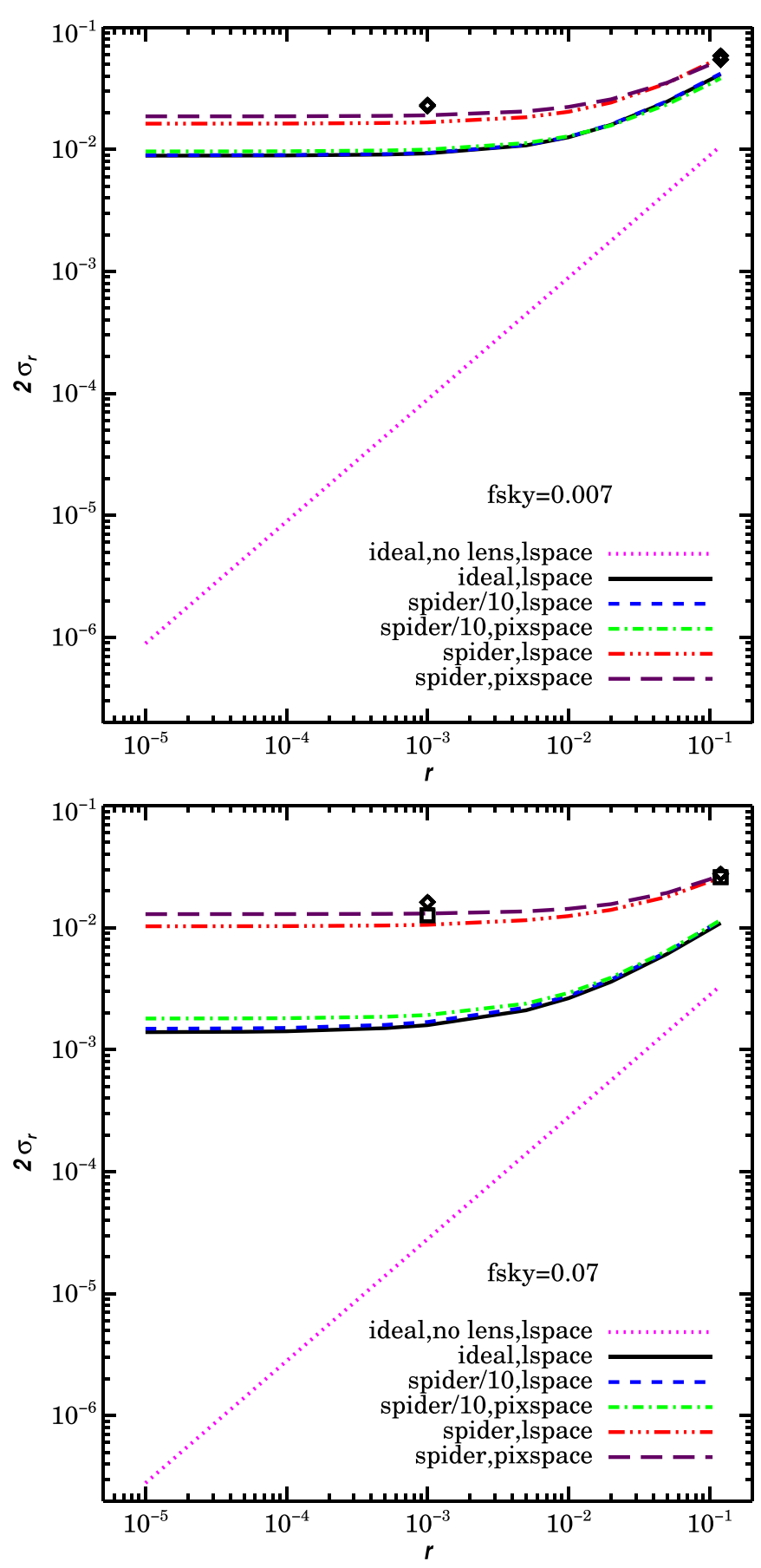

Figure 7. Curves show $2 \sigma_{r}$ as a function of $r_{\text {fid }}$ obtained from the Fisher matrix in $\ell$ and pixel-space for $f_{\text {sky }}=0.007$ (top) and 0.07 (bottom). The choices for the curves are meant to unravel the impact of the cosmic variance, lensing, instrument noise, and mode mixing on $\sigma_{r}$. The symbols show errors from the full likelihood calculated on a gridded $2 \mathrm{D}$ parameter space, and agree nicely for both pixel-space (squares) and $\ell$-space (diamonds).

(A color version of this figure is available in the online journal.)

is optimal for $r$ detection is awful for $\tau$ determination, for which all-sky is best.

\subsection{Results in $r-n_{s}$ Space}

In Figure 9, we have plotted the $r-n_{\mathrm{s}}$ contours for an $f_{\text {sky }}=0.08$ Spider-like experiment and for a full-sky Plancklike survey, with and without foregrounds, using the model discussed in Section 4.4. This shows almost no correlation 

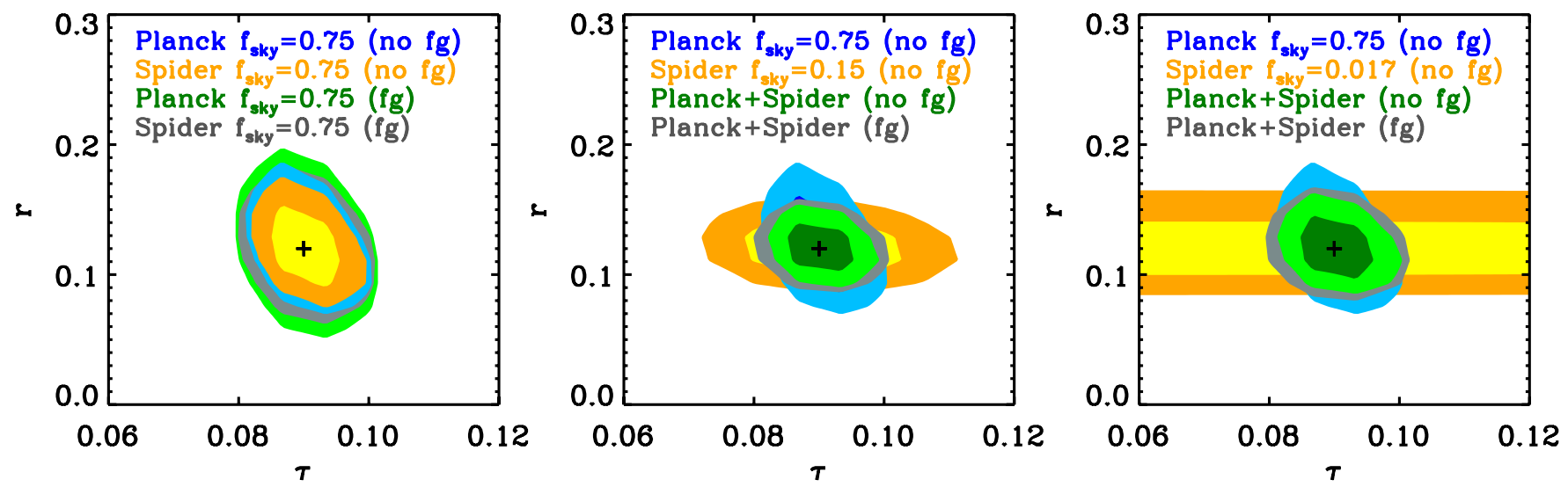

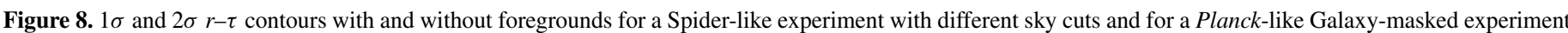

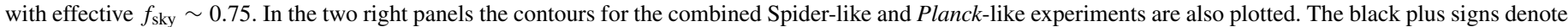

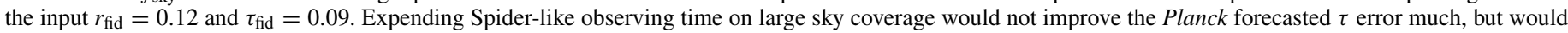
decrease the combined $r$ error, suggesting that the deep small-sky option is better.

(A color version of this figure is available in the online journal.)

between the two parameters for these experimental cases, as expected from the discussion in Section 4.3. It also shows the remarkable set of inflation constraints that may arise from Planck and Spider-like experiments.

\subsection{Results in $r-n_{t}$ Space}

Although detecting $r$ would provide an invaluable measure of the mean acceleration parameter (and energy scale) of inflation, we want more, the shape of the tensor power embodied in the tensor tilt $n_{\mathrm{t}}$. We explore this here in a 2D space by fixing $\tau, n_{\mathrm{s}}$ and the other cosmic parameters. Figure 10 shows the $2 \mathrm{D}$ contours for $r-n_{\mathrm{t}}$ with $r_{\mathrm{fid}}=0.12$, and fiducial tensor tilt $n_{\mathrm{t} \text {,fid }}=-0.0150$ satisfying the inflation consistency condition (Equation (1)). Alas, we see that $n_{\mathrm{t}}$ is hardly constrained by Spider-like and Planck-like experiments, no matter how large $f_{\text {sky }}$ is. To see whether a post-Planck deep all-sky experiment could modify this conclusion, we ran our analysis using the specification of a putative mid-cost $C M B P o l$ mission outlined in Baumann et al. (2009), using the frequency channels described in Table 1. There is of course improvement, and the COrE and PIXIE post-Planck missions would do better, but the relatively short $\Delta \ell \sim 150$ baseline precludes even an ideal experiment from providing a powerful test of inflation consistency.

\subsection{Breaking $r$ Up into $r_{X \beta}$-Shape Parameters: A Tensor Consistency Check}

Because $r$ is essentially a linear parameter (for given $A_{\mathrm{s}}$ ), we are effectively determining a single (very) broad bandpower amplitude multiplying a collection of fiducial $X$-template shapes $\mathcal{C}_{X \ell}^{(\mathrm{g})}$ given by the gravitational wave powers. It is natural to test this locked-in monolithic parameterization by introducing a collection of parameters $r_{X \beta}$ multiplying individual $X$ and $\ell$-band templates, i.e.,

$$
\begin{aligned}
\mathcal{C}_{E E \ell} & =\mathcal{C}_{E E \ell}^{(\mathrm{s})}+r_{E E \beta} \chi_{\beta}(\ell) \mathcal{C}_{E E \ell}^{(\mathrm{g})} \\
\mathcal{C}_{B B \ell} & =\mathcal{C}_{B B \ell}^{(\mathrm{lens})}+r_{B B \beta} \chi_{\beta}(\ell) \mathcal{C}_{B B \ell}^{(\mathrm{g})}
\end{aligned}
$$

Here $\mathcal{C}_{E E \ell}^{(\mathrm{s})}$ is the scalar part of $\mathcal{C}_{E E \ell}$, including lensing, and $\mathcal{C}_{B B \ell}^{\text {(lens) }}$ is the lensed $\mathrm{BB}$ power. The overall normalization is arranged so that $r_{X \beta}=r$ is the tensor consistency condition. The $\chi_{\beta}(\ell)$ 's are the $\beta$-windows. These can be taken to be top-hats satisfying a saturation property $\sum_{\beta} \chi_{\beta}(\ell)=1$ and an orthogonality property $\chi_{\beta}(\ell) \chi_{\beta}^{\prime}(\ell)=\delta_{\beta \beta^{\prime}}$ in bandpower work. The modes could also be quite overlapping as long as saturation and the $r_{X \beta}=r$ normalization are satisfied.

This is a reasonable path to finding the tensor bandpowers for $B B$ and $E E$ but, given the Section 4.6 result on $n_{\mathrm{t}}$, we will content ourselves with a 2D example using one $\ell$-band $\beta$ and two $X$ parameters, $r_{E E}$ and $r_{B B}$. For this study, we keep $A_{\mathrm{s}}$ fixed (cf. Sections 4.4 and 4.6). The contours in Figure 11 show the degree to which the tensor consistency encoded in the $r_{E E}=r_{B B}$ line, can be checked. The contours confirm the expectation that the $B$-modes are the most influential source of information about primordial tensor perturbations, since the large scalar contribution to $E E$ swamps the tiny tensor signal and inflates the error bars. Using checks like these for showing consistency have had a long history. In the first $E E$ polarization detection papers, the EE amplitude was shown to be consistent with the amplitude expected from $T T$ parameters (Kovac et al. 2002; Sievers 2004). In the first lensing detections in the TT power spectra, the deviations from lens-free results were shown to be consistent with expectations from the parameters determined from the primary TT data (Reichardt et al. 2009; Das et al. 2011b).

\subsection{Breaking $f_{\text {sky }}$ into Many Fields}

Using multiple (foreground-minimized) fields to make up a total $f_{\text {sky }}$ is an approach that has been advocated for groundbased strategies (e.g., for ABS; Essinger-Hileman et al. 2010). In Figure 12 we show the impact of splitting $f_{\text {sky }}$ into four patches, while keeping the total integration time and the instrument noise constant. One does not lose that much as long as the total probe is a few percent of the sky, a consequence of the broad single-patch $\sigma_{r}\left(f_{\text {sky }}\right)$ minimum. The number of polarizationforeground-clean patches is of course still to be determined. We also varied the patch geometry; e.g., for an $f_{\text {sky }} \sim 0.08$ rectangular region with $r_{\text {fid }}=0.12$, we get $2 \sigma_{r}=0.048$ without foregrounds, in good agreement with the cap result $2 \sigma_{r}=0.050$.

\section{SUMMARY AND CONCLUSIONS}

In this paper, we applied a full map-based likelihood analysis to multifrequency $Q-U$ polarization maps and $T$-maps of forecasted data to determine the posterior probability distribution of $r$. 

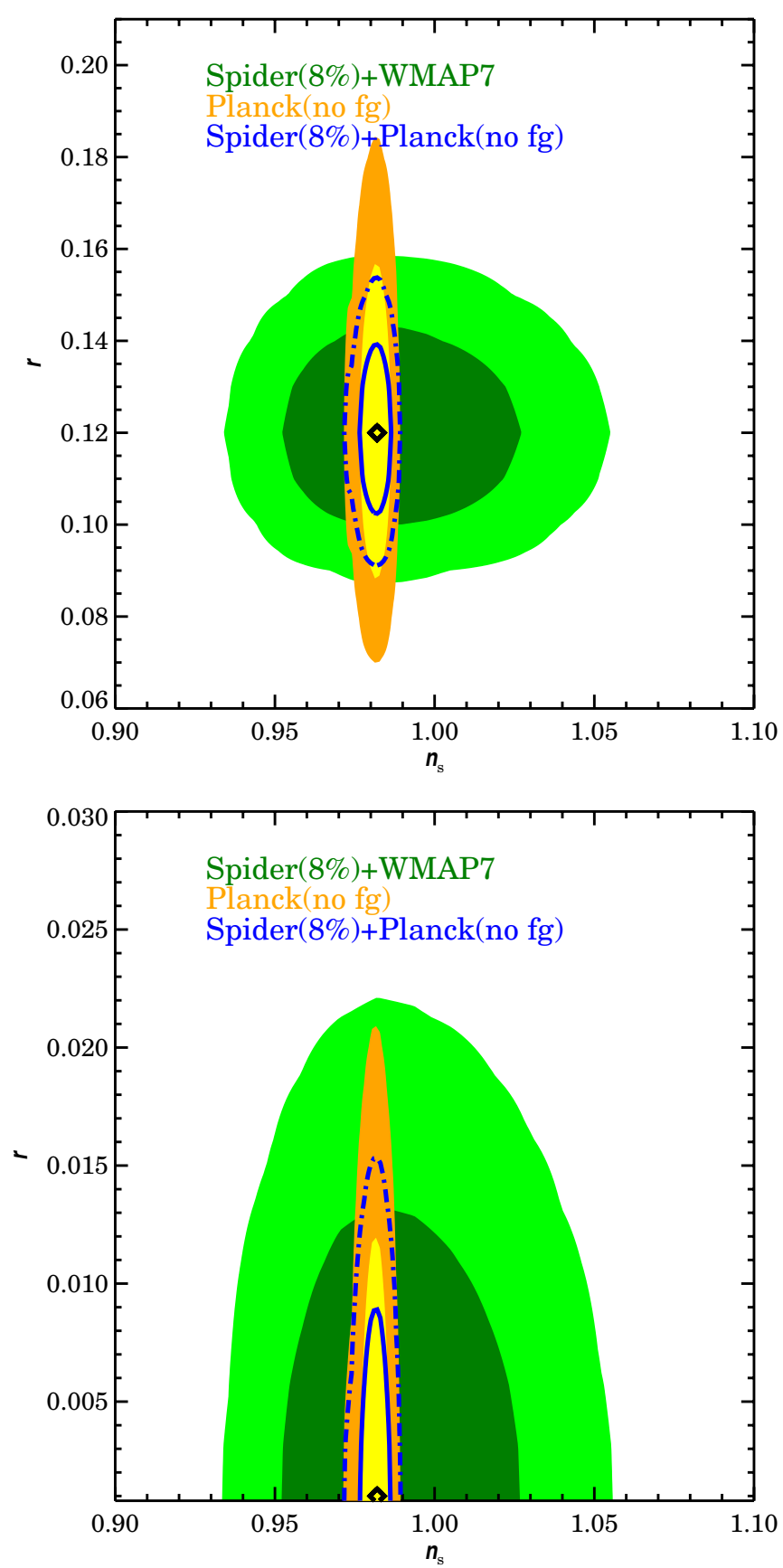

Figure 9. $r-n_{\mathrm{s}}$ contours for a Spider-like $\left(f_{\text {sky }} \sim 0.08\right)+W M A P 7$ experiment, contrasted with a Planck-like (Galaxy-masked $f_{\text {sky }} \sim 0.75$ ) experiment, assuming no foreground contamination, compared to the results for the combination of the two (the solid and dot-dashed blue curves). The $r-n_{\mathrm{s}}$ correlation has been ignored, as discussed in Section 4.3. We also explicitly verified this for the specific case of the Planck-like survey from post-processing the CosmoMC chains. The $r$ constraints are calculated by the numerical methods used throughout the paper. For the case of Spider+WMAP7, we assumed an asymmetric Gaussian likelihood for $n_{\mathrm{s}}$ with the widths coming from the lower and upper $1 \sigma_{n_{\mathrm{s}}}$ as measured by WMAP7 (http://lambda.gsfc.nasa.gov/product/ map/dr4/params/lcdm_sz_lens_tens_wmap7.cfm). For the $n_{\mathrm{s}}$ likelihood from the Planck-like case, CosmoMC chains (http://cosmologist.info/cosmomc/) were used to properly take into account the correlations of $n_{\mathrm{s}}$ with other cosmic parameters, which, unlike $r$, are non-negligible. The top has $r_{\text {fid }}=0.12$ and the bottom has 0.001 ; both have $n_{\mathrm{s} \text {, fid }}=0.98$. The plots indicate a possibly very rosy picture for constraining these two critical inflation parameters.

(A color version of this figure is available in the online journal.)

\subsection{Leakage Levels and Leakage Avoidance}

The map-based method discussed in this paper avoids the explicit linear $E-B$ decomposition of the polarization maps before doing the likelihood analysis and gives the best possible determination of $r$, provided that systematic errors are correctly modeled. For realistic cut-sky observations, we measured the level of $B B$ contamination from the inevitable mode-mixing from the much larger $E E$ power. In addition, there is leakage from instrumental effects, in particular with $T$ seeping into $Q$ and $U$, which has to be included in any approach. We have left the investigation of this issue to future work.

\subsection{Computational Feasibility of Exact Likelihoods}

It is often the case in CMB cosmology that the shear number of pixels precludes a direct full map-based likelihood procedure and necessiates an intermediate power spectrum determination before parameter estimation. However, for Spider and similar ground and balloon experiments targeting $r$, relatively low resolution and restricted sky coverage are all that is really needed for detection. The result is a total pixel number that allows computationally feasible inverse and determinant calculations of the large signal-plus-noise correlation matrices $\mathbf{C}_{\mathrm{t}}=\mathbf{C}_{\mathrm{n}}+\mathbf{C}_{\mathrm{s}}(q)$ - with contributions from both the parameterdependant signal covariance $\mathbf{C}_{\mathrm{s}}(q)$ and the generalized noise $\mathbf{C}_{\mathrm{n}}$, which includes uncertainties from the foreground subtraction as well as from instrumental and systematic noise in the maps

Map-based methods have had a long history, dating from the earliest CMB data sets (e.g., Bond \& Crittenden 2001). For example, they were used for COBE (Bond 1995), Saskatoon (Netterfield et al. 1997), BOOMERanG (Ruhl et al. 2003) and CBI (Myers et al. 2003) analyses. Often compression was used, e.g., to signal-to-noise eigenmodes (Bond 1995; Bond \& Crittenden 2001) or by coarse-grained gridding (Myers et al. 2003), to make the matrix manipulations tractable. With BOOMERanG, an important aspect was to make sure all issues regarding data-filtering, inhomogeneous and aspherical beams, transfer functions, striping, etc. were properly included. Invariably, a Monte Carlo simulator of each experiment has been built, in which simulated timestreams have as many effects from systematic and data processing as one can think of included.

\subsection{Matrix Estimation from Monte Carlo Noise and Signal Simulations and Relation to Master/XFaster}

In the estimation of the CMB power spectrum, the Master/XFaster approach uses Monte Carlo simulations to encode systematic and data processing effects of an experiment into isotropized $\ell$-space filters and rotationally symmetrized masks with the goal to relate the underlying $\ell$-space all-sky signal $\mathbf{C}_{\mathrm{s}, c X \ell}$ to the filtered cut sky signal (Hivon et al. 2002; Rocha et al. 2010, 2011; C. R. Contaldi et al. 2010, in preparation). Similarly an isotropized noise $\mathbf{C}_{\mathrm{n}, c X \ell}$ is determined by taking processed noise timestreams, creating maps with them, $Y_{\ell m}$ transforming them, then forming a quadratic average over noise samples. $J_{s}, \mathbf{C}_{\mathrm{n}, c X \ell}=\sum_{J_{s}, m}\left|a_{\mathrm{n} J_{s}, c X \ell m}\right|^{2} /\left[(2 \ell+1) N_{s}\right]$. $N_{s}$ is the number of noise samples. 

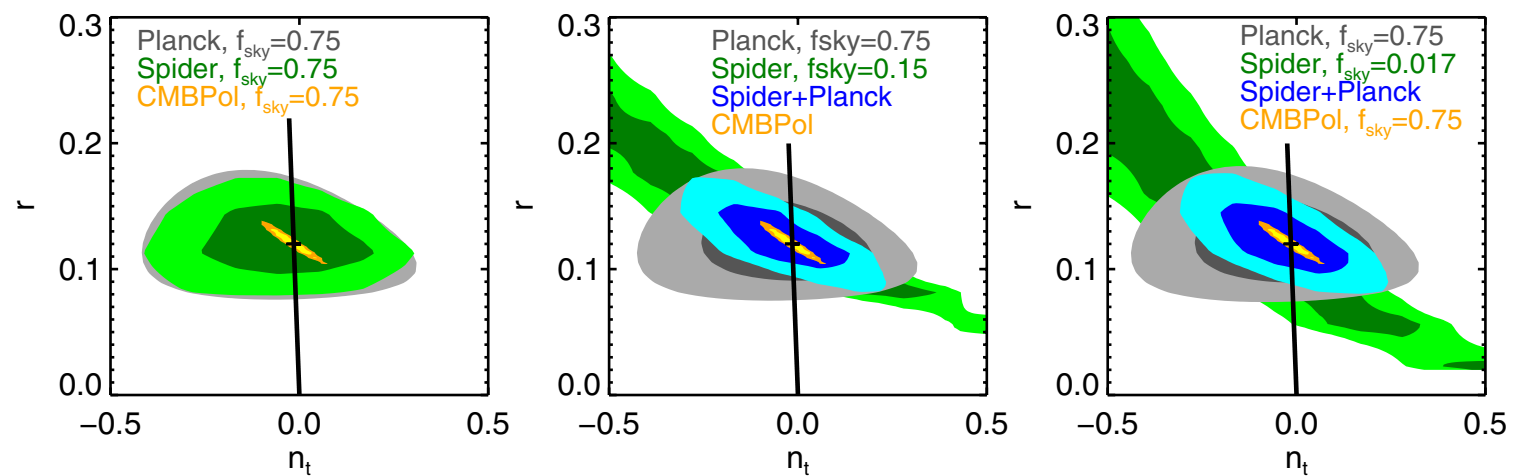

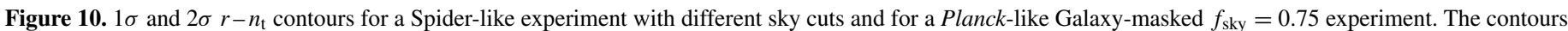

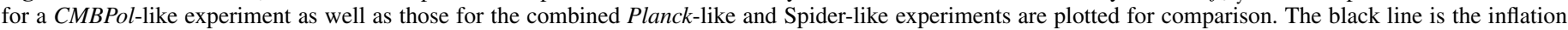
consistency line and the black plus sign is the fiducial input, $r=0.12$ and $n_{\mathrm{t}}=-0.015$. Even with this $C M B P o l$, inflation consistency is not that well tested.

(A color version of this figure is available in the online journal.)
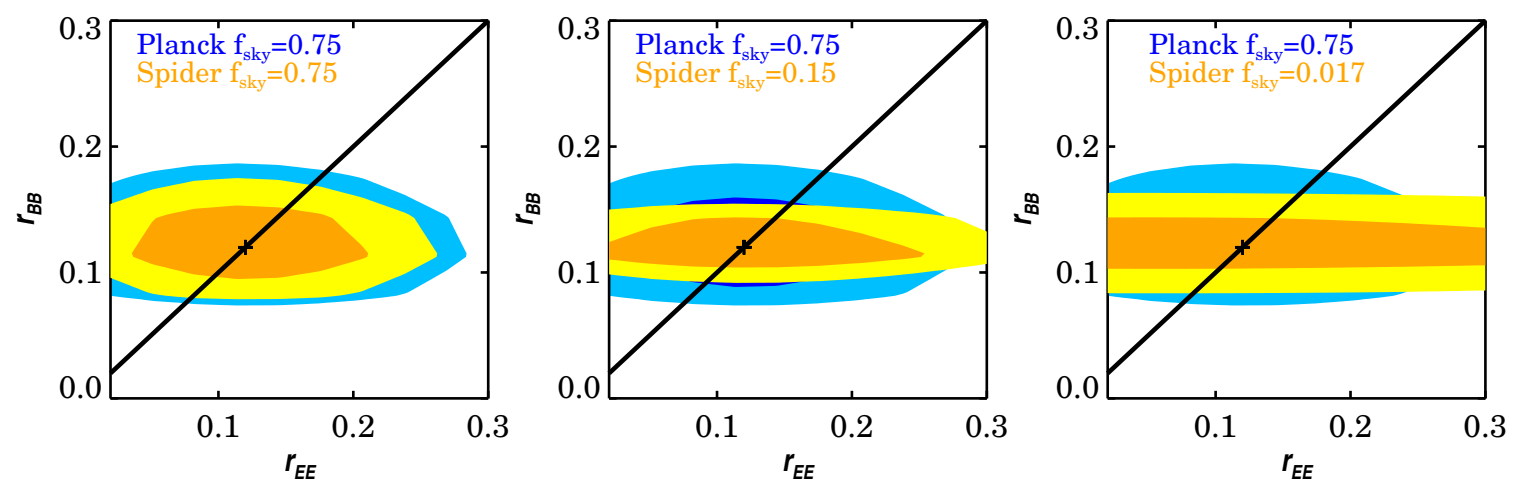

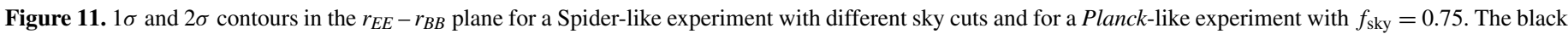

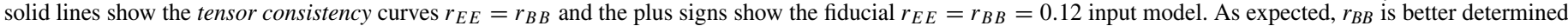
than $r_{E E}$ and this tensor consistency is not well tested.

(A color version of this figure is available in the online journal.)

Any operation that can be done for Master or XFaster can also be done to estimate the noise matrices, using noise sample sums. (Getting convergence of small off-diagonal components may require many samples.) Matrices have the advantage that they naturally allow for anisotropic and inhomogeneous components, in the noise maps-including striping effects - and in the beam maps and in the foreground maps. There are issues about optimal estimation of the generalized pixel-pixel matrices that one would like to tune, but there are no fundamental obstacles to making the $\mathbf{C}_{\mathrm{n}}$ and $\mathbf{C}_{\mathrm{s}}$ matrices highly accurate for parameter estimation.

WMAP used a map-based likelihood for low $\ell$, connected to an isotropized $\ell$-space likelihood covering the high $\ell$ 's (Hinshaw et al. 2007). Planck is doing the same. We expect such a hybridized likelihood code will also be used for Spider-like experiments for routine parameter estimation, even though we think one can get away with a full map-based likelihood code.

If simulated timestreams are used for $\mathbf{C}_{n}$ and $\mathbf{C}_{\mathrm{s}}$ estimation, generalized pixels may prove preferable to the usual spatial pixels. The Cosmic Background Imager (CBI; Myers et al. 2003; Sievers 2004) used the reciprocal space pixels for the primary construction, rather natural for an interferometry experiment where the timestream analog is a set of visibilities. ACT (Das et al. 2011a) and QUaD (Pryke et al. 2009) also have done their power spectrum estimation in the Fourier transform space of spatial maps.

\subsection{The CBIpol Approach as a Guide for Small Deep-sky Analyses}

The use of map-based likelihood codes for $r$ estimation does not mean that $E$ and $B$ maps will not be constructed, just that parameters would not be extracted from them. The CBI example of how such $E$ and $B$ maps were made and used, and why bandpower and parameter estimations did not use $E$ and $B$ maps serves as a paradigm for how things could proceed for Spider-like data (Myers et al. 2003; Sievers et al. 2007). The CBI data were compressed (via a GRIDR code) onto a discrete (reciprocal) lattice of wavenumbers by projecting measured interferometer visibilities onto a gridded 2D K-space. A direct unitary transformation takes such a basis of "momentum" modes into a basis of spatial modes in real space where $Q-U$ is a more appropriate representation. An important point is that the polarization map estimators evaluated on the discrete wavenumbers of the lattice are linear combinations of the continuous wavenumbers. This leads to the mode coupling in the finite maps, particularly to an $E-B$ mixing.

In the lattice representation, the resulting size of the correlation matrices for CBI was quite tractable for direct inversion and the full likelihood was evaluated to determine bandpowers for $T T, E E, B B$ and $T E$, without separation of the Fourier maps into $E$ and $B$.

An optimal linear map reconstruction of $E$ and $B$ was done for visualization purposes, with real-space and 


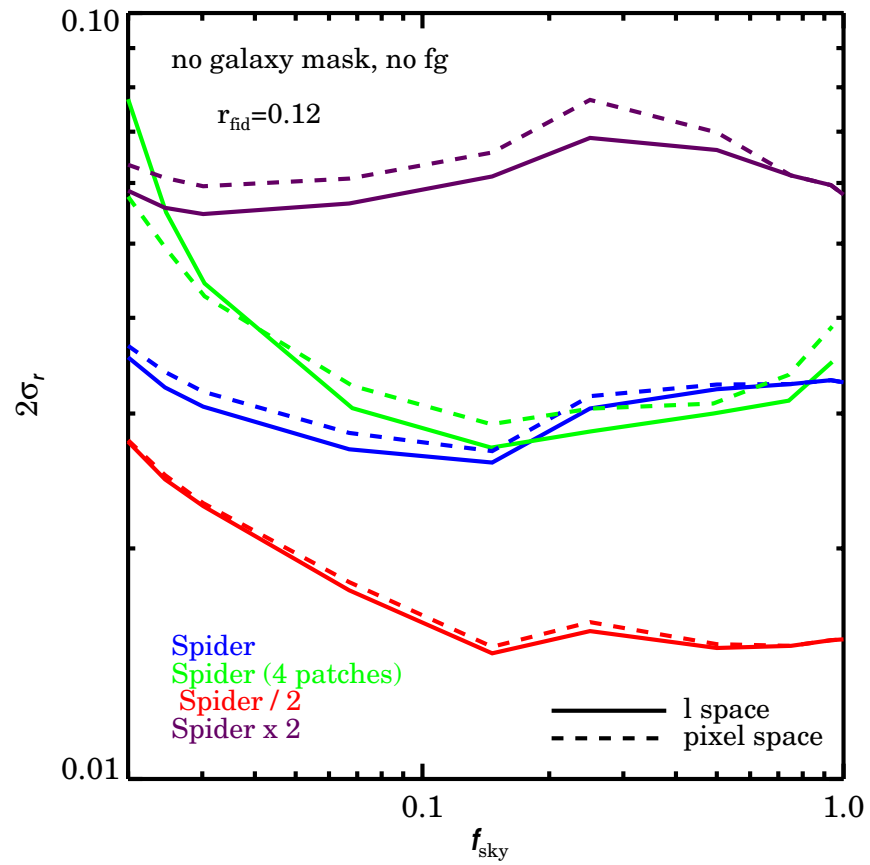

Figure 12. When one patch covering $f_{\text {sky }}$ is broken up into four $f_{\text {sky }} / 4$ cappatches, but the noise and observing time remain constant, the ( $\tau$-marginalized) $r$-errors remain similar except at very small $f_{\text {sky }}$. We also show that factors of two changes in the noise swamp this effect. The calculations were done with $r_{\text {fid }}=0.12$ in the pixel-space, except for the highest sky coverages where the pixel and $\ell$-space analysis are in excellent agreement. The effect of foreground contamination and Galaxy cut has not been taken into account here.

(A color version of this figure is available in the online journal.)

momentum-space maps showing the CBI $E$ and $B$ Wienerfiltered means, accompanied by a few maps showing typical fluctuation maps about the mean maps. These were contour maps, in contrast to the usual polarization plots which consist of headless vectors of the length of polarization degree, $\sqrt{Q^{2}+U^{2}}$, tilted at an $\operatorname{angle} \arctan (U / Q) / 2$.

For Spider-like bolometer-based experiments for which the raw data are bolometer time streams from which $Q-U$ maps are constructed, the compression step leads to tractable matrices as in the CBIpol case, although in the first instance the pixelization choice may be in real space rather than in wavenumber space or in a generalized-pixel space. Just as with CBIpol, parameters and bandpowers would be determined with direct likelihood calculations, yet Wiener-filtered $E$ and $B$ maps would still be made for visualization.

\subsection{Exact 2D Likelihood Computation}

Given the matrix construction method, we determined the posterior probabilities on reduced 2D-grids consisting of $r$ and one other cosmic parameter, in many cases the Thomson scattering depth to reionization, $\tau$. The grid could be extended to higher dimensions, as they were in early $\mathrm{CMB}$ analyses of COBE (Bond 1995), BOOMERanG (Ruhl et al. 2003), CBI (Myers et al. 2003) and ACBAR (Kuo et al. 2007). More efficiently, MCMC chains could be used to explore the posterior probability surface. Since, as we have shown, $r$ is relatively weakly correlated with the other standard cosmic parameters, our use of a reduced dimensionality is accurate. We targeted $\tau$ for a second parameter because of its importance for the reionization bump in $B B$ which is picked by large $f_{\text {sky }}$ experiments such as Planck. However, it too is weakly correlated for Spider-like experiments probing modest $f_{\text {sky }}$. We showed that as long as the input value $r_{\text {fid }}$ is reasonably larger than the error $\sigma_{r}$, e.g., $\sim 0.1$, $r_{\text {fid }}$ can be well recovered by our methods.

\subsection{The Inflation and Tensor Consistency Checks}

We have used $r$ and $n_{\mathrm{t}}$ for our reduced 2D parameter space to see how well the inflation consistency condition, $n_{\mathrm{t}} \approx-r / 8$, can be tested. For example, with $r_{\text {fid }}=0.12$ and the consistency value $n_{\mathrm{t}, \text { fid }}=-0.015$, we obtain $2 \sigma_{r} \approx 0.036$ and $2 \sigma_{n_{\mathrm{t}}} \approx 0.28$. The large $1 \sigma$ error on $n_{\mathrm{t}}$ is what one might have expected given the relatively small $\ell$-baseline (reminiscent of the \pm 0.2 limit on $n_{\mathrm{s}}$ from the even smaller baseline COBE DMR data). Thus, although breaking up $r$ into bands will be useful, the $n_{\mathrm{t}}$ slope that follows will not be powerful enough to test consistency. With CMBPol and at $N_{\text {side }}=512$, the errors are $2 \sigma_{r} \approx 0.014$ and $2 \sigma_{n_{\mathrm{t}}} \approx 0.07$, which are still too large. A more prosaic internal consistency check was done to show that what one thinks is $r$ from the total $B B$ agrees with what one gets from the lesstensor-sensitive total $E E$.

\subsection{Relation to Planck}

We based our Planck-like case on the Blue Book detector specifications. The actual in-flight performance is quite similar (Planck HFI Core Team et al. 2011; Mennella et al. 2011). What will emerge from the actual Planck polarization analysis may be quite different from the simplified foreground-free $2 \sigma_{r}\left(f_{\text {sky }}=0.75\right) \sim 0.015$ forecast of white experimental noise and with no systematics. This relies on the $B B$ reionization bump being picked up, but the required low $\ell$ 's are especially susceptible to the foreground-subtraction residuals $\left(2 \sigma_{r}\left(f_{\text {sky }}=\right.\right.$ $0.75) \sim 0.05$ for a model of well-subtracted foregrounds of known residual) and systematic effects. Some of the issues are described in Efstathiou et al. (2009). Irrespective of how well Planck wrestles with the low $\ell$ issues, it will be able to analyze many patches within the $75 \%$ of the sky, rank-ordered by degree of foreground contamination. Although such a procedure would lose the reionization bump, robustness to foreground threshold variation of any $r$-detection could be well demonstrated. Apart from its many other virtues, Planck should be very good for this.

\subsection{Relation to Spider}

The same strategy of using many fields with the lowest foregrounds to make up the total $f_{\text {sky }}$ may also prove useful for Spider-like experiments (such as the ground-based ABS). We showed that splitting $f_{\text {sky }}$ into four patches with fixed integration time and the instrument noise results in only a small loss in $r$-sensitivity because $\sigma_{r}\left(f_{\text {sky }}\right)$ has a relatively wide single-patch minimum. How many polarization-foreground-clean patches there are is still to be determined.

Although the specifications we chose for "Spider-like" were motivated by a bolometer array experiment feasible with current technology, our forecasts should not be taken as realistic mocks of the true Spider which is under development, and for which a number of campaigns are envisaged (see the footnote under Spider-like in Table 1). The techniques used here have, however, already been applied in Spider forecast papers using more realistic statistically inhomogeneous noise, scanning strategies and observational durations, e.g., in Filippini et al. (2010) and Fraisse et al. (2013). On $f_{\text {sky }} \sim 0.1, r_{\text {fid }}=0.01$ simulations, we compared the Fraisse et al. (2013) non-uniform noise modulated spatially by the scanning strategy's number-of-hits-per-pixel with uniform white noise with the same integrated noise power. 
Although the deviation in the standard deviation of the noise rms was about a factor of two times the mean noise $r m s$, with largest impact near the scanning boundaries, we found very similar results for the posterior, showing that this paper's conclusions are insensitive to our use of uniform white noise. (Of course the foreground noise radically alters the whiteness, and this of course has been included by us, but only in a statistically isotropic way-the Galactic latitude dependence breaks this isotropy just as the pixel hits do.) In Section 4.4, we showed that in the absence of foregrounds our Spider-like case could achieve $2 \sigma_{r} \approx 0.02$ over a broad range of $f_{\text {sky }}$.

We presented Figure 1 in the introduction as a summary of the current and future $r$-posteriors. The forecasted likelihood curves were made with the numerical codes described here, for the Spider-like case (the default experiment of this paper), for the Spider experiment labeled "Spider" in the plot (Fraisse et al. 2013), and for a more ambitious campaign of Spider, labeled "SCIP." We see that the performance of the experiment with Spider-like specifications used in this paper is very close to the actual Spider. A different foreground model used in Fraisse et al. (2013) for $f_{\text {sky }} \sim 0.1$ led to a similar $\sim 50 \%$ error degradation.

\subsection{History and Forecasts of $r$ Constraints}

When the large angle CMB anisotropies were first detected with COBE DMR (Smoot et al. 1992), the broadband TT power amplitude $(\ell \lesssim 20)$, with wavenumbers $k^{-1} \gtrsim 1000 \mathrm{Mpc}$, was related to the linear density power spectrum amplitude at the radically different $k^{-1} \sim 6 \mathrm{Mpc}$ scale, assuming a nearly scaleinvariant primoridial spectrum: $\sigma_{8} \approx 0.85 e^{-(\tau-0.1)} / \sqrt{1+0.6 r} \times$ $1_{-0.6}^{0.7}$ for typical $\Lambda$ CDM parameters popular in mid-1990s, $\Omega_{\Lambda} \sim 2 / 3, h \sim 0.7$ (Bond 1996), rather similar to the values now. Requiring $\sigma_{8}>0.7$ to get reasonable cluster abundances at zero redshift - a venerable cosmological requirement from the 1980s-gives a rough constraint on $r$ from the COBE data in conjunction with large scale structure (LSS) data: $2 \sigma_{r}<1$ for current $\tau$ values - but $\tau$ only had an upper limit until WMAP1 (Spergel et al. 2003), with a more accurate determination waiting until WMAP3 (Spergel et al. 2007).

The first 2003 WMAP constraint on $r$ from $T T$ and $T E$ CMB-only data (with weak priors) was $2 \sigma_{r}<0.81$, reducing to $2 \sigma_{r}<0.62$ with the WMAP3 TT, TE, and EE data, and other $T T$ CMB data available in 2005 . It decreased to 0.31 with the LSS data of the time (MacTavish et al. 2006). The most recent $r$-constraint from the low $\ell$ amplitude and shape of the $T T$ and $E E$ spectra from WMAP9+ACT+SPT is the upper limit $2 \sigma_{r} \sim 0.17$, reducing to 0.12 when LSS is added (Figure 1; Hinshaw et al. 2012).

Making a further leap awaits an effective $B$-mode constraint. The current best constraint on $r$ from the measurements of the $B$-mode amplitude comes from the QUIET experiment with $2 \sigma_{r}<2.8$ (QUIET Collaboration et al. 2012). As we have seen, Planck can give 0.015-0.05, Spider 0.014-0.02. The COrE satellite proposal (The COrE Collaboration 2011) suggests better than a $3 \sigma$ detection could be made for $r_{\text {fid }}$ above 0.001 with bolometer arrays in space. The PIXIE satellite proposal (Kogut et al. 2011) claims $2 \sigma_{r} \approx 4 \times 10^{-4}$ is achievable with Fourier transform spectrometry. We apply our methods to CMBPol specifications (Baumann et al. 2009). The two cases in Figure 1 show what a (very small) detection with $r_{\text {fid }}=0.001\left(2 \sigma_{r} \approx 4 \times 10^{-4}\right)$ and a non-detection with $r_{\text {fid }}=0.0001\left(2 \sigma_{r} \approx 1.2 \times 10^{-4}\right)$ would look like. If $r_{\text {fid }}$ is as large as 0.12 , as in the simple $m^{2} \phi^{2}$ chaotic inflation, we get $2 \sigma_{r} \approx 0.015$ (and $2 \sigma_{n_{\mathrm{t}}} \approx 0.07$ encompassing the consistency input of $\left.n_{\mathrm{t}}=-0.015\right)$. For a noiseless all-sky experiment, hence with errors from cosmic variance only, we get $2 \sigma_{r} \approx 10^{-4}$ for $N_{\text {side }}=128$ for tiny $r_{\text {fid }}$. It is unclear at this time how much inexact foreground subtraction and lensing noise will limit $r$ determinations in these ideal cases.

\subsection{The 1D Shannon Entropy of $r$}

We have described another way to cast the improvements expected in $r$-estimation as experiments attain higher and higher sensitivity, the marginalized 1D Shannon entropy for $r, \Delta S_{1 \mathrm{f}}(r)$. This measures the (phase-space) volume of $r$-space that the measurement allows. It is obtained by direct integration over the normalized 1D likelihood for $r$, with all non-Gaussian features in the likelihood properly included. We have found in practice that $\Delta S_{1 \mathrm{f}}(r) \approx \Delta \ln \left[\sigma_{r} \sqrt{2 \pi}\right]$, with $\sigma_{r}$ determined by the forced Gaussianization described in the paper, works quite well, so in a way we are just restating the error improvements in the information theoretic language of bits.

We use the current WMAP9+ACT+SPT $T T, T E$ and $E E$ + LSS $2 \sigma_{r} \sim 0.12$ constraint (Hinshaw et al. 2012) for our baseline. The first WMAP constraint in 2003 (Spergel et al. 2003), with $\Delta S_{1 \mathrm{f}}(r)=2.7$ bits had, of course, higher information entropy. Here, as in the Abstract, we have translated from nats to bits. The asymptotic perfect noiseless all-sky experiment gives (the somewhat $r$-dependent) $\Delta S_{1 \mathrm{f}}(r) \approx-10$ bits, the limit on obtainable knowledge from the CMB. The proposed post-Planck COrE, PIXIE and CMBPol-like experiments claim about -8 bits. For the Spider-like experiments forecasted here, the foreground-free decrease is -3.1 bits (and -2.6 bits with a $95 \%$ effective component separation). Thus balloon-borne and ground-based experiments with large arrays making deep surveys focusing on a relatively clean few percent of the sky yield tensor information at least comparable to shallow and wide surveys and are a powerful step toward a near-perfect deep and wide satellite future.

We thank our many Spider, ABS, and Planck collaborators for many stimulating discussions about the experimental assault on CMB tensor mode detection. We thank William C. Jones for his helpful comments on the text. We thank Marc Antoine Miville Deschênes for advice and aid on foregrounds. Support from NSERC, the Canadian Institute for Advanced Research, and the Canadian Space Agency (for PlanckHFI and Spider work) is gratefully acknowledged. Part of the research described in this paper was carried out at the Jet Propulsion Laboratory, California Institute of Technology, under a contract with the National Aeronautics and Space Administration. The large matrix computations were performed using the SciNET facility at the University of Toronto. Some of the results in this paper have been derived using the HEALPix package (Górski et al. 2005), http://healpix.jpl.nasa.gov.

\section{REFERENCES}

Bardeen, J. M., Steinhardt, P. J., \& Turner, M. S. 1983, PhRvD, 28, 679

Baumann, D., Jackson, M. G., Adshead, P., et al. 2009, in AIP Conf. Proc. 1141, CMB Polarization Workshop: Theory and Foregrounds: CMBPol Mission Concept Study, ed. S. Dodelson, D. Baumann, A. Cooray et al. (Melville, NY: AIP), 10

Bond, J. R. 1995, PhRvL, 74, 4369

Bond, J. R. 1996, in Cosmology and Large Scale Structure, ed. R. Schaeffer, J. Silk, M. Spiro, \& J. Zinn-Justin (Amsterdam: Elsevier), 469

Bond, J. R., \& Crittenden, R. 2001, in NATO ASIC Proc. 565: Structure Formation in the Universe, ed. R. G. Crittenden \& N. G. Turok (Dordrecht: Kluwer), 241 
Bond, J. R., Jaffe, A. H., \& Knox, L. 1998, PhRvD, 57, 2117

Bunn, E. F. 2011, PhRvD, 83, 083003

Bunn, E. F., Zaldarriaga, M., Tegmark, M., \& de Oliveira-Costa, A. 2003, PhRvD, 67, 023501

Challinor, A., \& Chon, G. 2005, MNRAS, 360, 509

Chiang, H. C., Ade, P. A. R., Barkats, D., et al. 2010, ApJ, 711, 1123

Chon, G., Challinor, A., Prunet, S., Hivon, E., \& Szapudi, I. 2004, MNRAS, 350,914

Chuss, D. T., Ade, P. A. R., Benford, D. J., et al. 2010, SPIE, 7741

Cooray, A., \& Kesden, M. 2003, NewA, 8, 231

Cooray, A., Melchiorri, A., \& Silk, J. 2003, PhLB, 554, 1

Das, S., Marriage, T. A., Ade, P. A. R, et al. 2011a, ApJ, 729, 62

Das, S., Sherwin, B. D., Aguirre, P., et al. 2011b, PhRvL, 107, 021301

de Bernardis, P., Ade, P. A. R., Bock, J. J., et al. 2000, Natur, 404, 955

Delabrouille, J., Betoule, M., Melin, J.-B., et al. 2012, arXiv:1207.3675

Dodelson, S. 2003, Modern Cosmology (1st ed.; Amsterdam: Academic)

Dunkley, J., Calabrese, E., Sievers, J., et al. 2013, arXiv:1301.0776

Efstathiou, G., \& Bond, J. R. 1986, MNRAS, 218, 103

Efstathiou, G., Gratton, S., \& Paci, F. 2009, MNRAS, 397, 1355

Essinger-Hileman, T., Appel, J. W., Beall, J. A., et al. 2010, arXiv:1008.3915

Filippini, J. P., Ade, P. A. R., Amiri, M., et al. 2010, SPIE, 7741, 77411N

Fraisse, A. A., Ade, P. A. R., Amiri, M., et al. 2013, JCAP, 4, 47

Gold, B., Odegard, N., Weiland, J. L., et al. 2011, ApJS, 192, 15

Górski, K. M., Hivon, E., Banday, A. J., et al. 2005, ApJ, 622, 759

Grain, J., Tristram, M., \& Stompor, R. 2009, PhRvD, 79, 123515

Guth, A. H., \& Pi, S.-Y. 1985, PhRvD, 32, 1899

Hansen, F. K., \& Górski, K. M. 2003, MNRAS, 343, 559

Hawking, S. W. 1982, PhLB, 115, 295

Hinshaw, G., Larson, D., Komatsu, E., et al. 2012, ApJS, submitted (arXiv:1212.5226)

Hinshaw, G., Nolta, M. R., Bennett, C. L., et al. 2007, ApJS, 170, 288

Hivon, E., Górski, K. M., Netterfield, C. B., et al. 2002, ApJ, 567, 2

Kallosh, R., \& Linde, A. 2010, JCAP, 11, 011

Kamionkowski, M., Kosowsky, A., \& Stebbins, A. 1997, PhRvD, 55, 7368

Kodama, H., \& Sasaki, M. 1984, PThPS, 78, 1

Kodama, H., \& Sasaki, M. 1986, IJMPA, 1, 265

Kofman, L. A., \& Linde, A. D. 1987, NuPhB, 282, 555

Kogut, A., Fixsen, D. J., Chuss, D. T., et al. 2011, JCAP, 7, 25

Kolb, E. W., \& Turner, M. S. 1990, The Early Universe (1st ed.; New York: Addison-Wesley)

Kovac, J. M., Leitch, E. M., Pryke, C., et al. 2002, Natur, 420, 772

Kuo, C. L., Ade, P. A. R., Bock, J. J., et al. 2007, ApJ, 664, 687

Lange, A. E., Ade, P. A., Bock, J. J., et al. 2001, PhRvD, 63, 042001

Larson, D., Dunkley, J., Hinshaw, G., et al. 2011, ApJS, 192, 16

Leach, S. M., Cardoso, J.-F., Baccigalupi, C., et al. 2008, A\&A, 491, 597

Lewis, A., Challinor, A., \& Turok, N. 2002, PhRvD, 65, 023505

Linde, A. D. 1983, PhLB, 129, 177

Linde, A. D. 1984, JETPL, 40, 1333

Lyth, D. H. 1997, PhRvL, 78, 1861

MacKay, D. J. 2003, Information Theory, Inference, and Learning Algorithms (1st ed.; Cambridge: Cambridge Univ. Press)
MacTavish, C. J., Ade, P. A. R., Bock, J. J., et al. 2006, ApJ, 647, 799

McAllister, L., Silverstein, E., \& Westphal, A. 2010, PhRvD, 82, 046003

Mennella, A., Butler, R. C., Curto, A., et al. 2011, A\&A, 536, A3

Montroy, T. E., Ade, P. A. R., Bock, J. J., et al. 2006, ApJ, 647, 813

Mukhanov, V. F. 2005, Physical Foundations of Cosmology (1st ed.; Cambridge: Cambridge Univ. Press)

Mukhanov, V. F., \& Chibisov, G. V. 1981, JETPL, 33, 532

Myers, S. T., Contaldi, C. R., Bond, J. R., et al. 2003, ApJ, 591, 575

Netterfield, C. B., Devlin, M. J., Jarolik, N., Page, L., \& Wollack, E. J. 1997, ApJ, 474, 47

O’Dea, D. T., Ade, P. A. R., Amiri, M., et al. 2011, ApJ, 738, 63

O'Dea, D. T., Clark, C. N., Contaldi, C. R., \& MacTavish, C. J. 2012, MNRAS, 419, 1795

Okamoto, T., \& Hu, W. 2002, PhRvD, 66, 063008

Page, L., Hinshaw, G., Komatsu, E., et al. 2007, ApJS, 170, 335

Peebles, P. J. E. 1987, Natur, 327, 210

Piacentini, F., Ade, P. A. R., Bock, J. J., et al. 2006, ApJ, 647, 833

Planck HFI Core Team, et al. 2011, arXiv:1101.2039

Pryke, C., Ade, P., Bock, J., et al. 2009, ApJ, 692, 1247

QUIET Collaboration, Araujo, D., Bischoff, C., et al. 2012, ApJ, 760, 145

QUIET Collaboration, Bischoff, C., Brizius, A., et al. 2010, arXiv:1012.3191

Reichardt, C. L., Ade, P. A. R., Bock, J. J., et al. 2009, ApJ, 694, 1200

Reichborn-Kjennerud, B., Aboobaker, A. M., Ade, P., et al. 2010, SPIE, 7741, $7411 \mathrm{C}$

Rocha, G., Contaldi, C. R., Bond, J. R., \& Górski, K. M. 2011, MNRAS, 414, 823

Rocha, G., Contaldi, C. R., Colombo, L. P. L., et al. 2010, arXiv e-prints

Ruhl, J. E., Ade, P. A. R., Bock, J. J., et al. 2003, ApJ, 599, 786

Sheehy, C. D., Ade, P. A. R., Aikin, R. W., et al. 2010, SPIE, 7741, 77411R

Sievers, J. L. 2004, PhD thesis, California Institute of Technology, CA, USA

Sievers, J. L., Achermann, C., Bond, J. R., et al. 2007, ApJ, 660, 976

Sievers, J. L., Bond, J. R., Cartwright, J. K., et al. 2003, ApJ, 591, 599

Sievers, J. L., Mason, B. S., Weintraub, L., et al. 2009, arXiv:0901.4540

Smith, K. M. 2006, PhRvD, 74, 083002

Smith, K. M., Cooray, A., Das, S., et al. 2008, arXiv:0811.3916

Smith, K. M., \& Zaldarriaga, M. 2007, PhRvD, 76, 043001

Smoot, G. F., Bennett, C. L., Kogut, A., et al. 1992, ApJL, 396, L1

Spergel, D. N., Bean, R., Doré, O., et al. 2007, ApJS, 170, 377

Spergel, D. N., Verde, L., Peiris, H. V., et al. 2003, ApJS, 148, 175

Starobinsky, A. A. 1982, PhLB, 117, 175

Story, K. T., Reichardt, C. L., Hou, Z., et al. 2012, arXiv:1210.7231

Szapudi, I., Prunet, S., \& Colombi, S. 2001, arXiv:astro-ph/0107383

Tegmark, M., \& de Oliveira-Costa, A. 2001, PhRvD, 64, 063001

The COrE Collaboration. 2011, arXiv:1102.2181

The Polarbear Collaboration, Errard, J., Ade, P. A. R., et al. 2010, arXiv: 1011.0763

Weinberg, S. 2008, Cosmology (1st ed.; Oxford: Oxford Univ. Press)

Wraith, D., Kilbinger, M., Benabed, K., et al. 2009, PhRvD, 80, 023507 Zaldarriaga, M. 2000, PhRvD, 62, 063510

Zaldarriaga, M., \& Seljak, U. 1997, PhRvD, 55, 1830

Zaldarriaga, M., \& Seljak, U. 1998, PhRvD, 58, 023003 